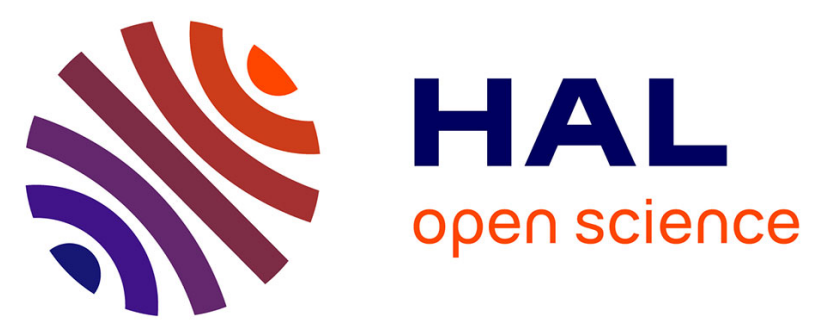

\title{
Synthesis of metathesis catalysts with fluorinated unsymmetrical N,N'-diaryl imidazoline-based NHC ligands
}

S.M. Masoud, M.A. Topchiy, A.S. Peregudov, Thierry Roisnel, Pierre H. Dixneuf, Christian Bruneau, S.N. Osipov

\section{To cite this version:}

S.M. Masoud, M.A. Topchiy, A.S. Peregudov, Thierry Roisnel, Pierre H. Dixneuf, et al.. Synthesis of metathesis catalysts with fluorinated unsymmetrical N,N'-diaryl imidazoline-based NHC ligands. Journal of Fluorine Chemistry, 2017, 200, pp.66-76. 10.1016/j.jfluchem.2017.06.004 . hal-01581217

\section{HAL Id: hal-01581217 \\ https://hal-univ-rennes1.archives-ouvertes.fr/hal-01581217}

Submitted on 4 Sep 2017

HAL is a multi-disciplinary open access archive for the deposit and dissemination of scientific research documents, whether they are published or not. The documents may come from teaching and research institutions in France or abroad, or from public or private research centers.
L'archive ouverte pluridisciplinaire HAL, est destinée au dépôt et à la diffusion de documents scientifiques de niveau recherche, publiés ou non, émanant des établissements d'enseignement et de recherche français ou étrangers, des laboratoires publics ou privés. 


\section{Synthesis of Metathesis Catalysts with Fluorinated Unsymmetrical $N, N$ '-Diaryl Imidazoline-based NHC ligands}

Salekh M. Masoud ${ }^{\mathrm{a}, \mathrm{b}}$, Maxim A. Topchiy ${ }^{\mathrm{a}}$, Alexander S. Peregudov ${ }^{\mathrm{a}}$, Thierry Roisnel ${ }^{\mathrm{c}}$, Pierre H.

Dixneuf $^{\mathrm{b}}$, Christian Bruneau ${ }^{\mathrm{b} *}$, and Sergey N. Osipov ${ }^{\mathrm{a} *}$

a A.N. Nesmeyanov Institute of Organoelement compounds, Russian Academy of Sciences, Vavilov str. 28, 119991, Moscow, Russia, e-mail: osipov@ineos.ac.ru

b UMR 6226 CNRS-Université de Rennes 1, Institut des Sciences Chimiques de Rennes, Organométalliques: Matériaux et Catalyse, Centre for Catalysis and Green Chemistry, Campus de Beaulieu, 35042 Rennes, France, e-mail: christian.bruneau@univ-rennes1.fr

${ }^{\mathrm{c}}$ UMR 6226 CNRS-Université de Rennes 1, Institut des Sciences Chimiques de Rennes, Centre de Diffractométrie X, Campus de Beaulieu, 35042 Rennes, France.

Highlights

- Fluorinated unsymmetrical N,N'-diaryl imidazolinium salts as new NHC ligand precursors for olefin metathesis catalysts.

- Synthesis of new ruthenium carbene complexes.

- Investigation of catalytic activity of new fluorinated complexes in RCM and CM reactions.

Abstract: New olefin metathesis catalysts bearing unsymmetrical fluorinated NHC ligands with hexafluoroisopropylmethoxy group in ortho-positions of $N$-aryl-substituent have been synthesized. The effects of mono-ortho-arylsubstitution and the replacement of para-methyl $\mathrm{N}$-aryl group with more electron-donating methoxy group in unsymmetrical fluorinated NHC ligand on the activity of complexes have been evaluated.

\section{Introduction}

Keywords: fluorinated NHC; ; ; , ruthenium complexes, olefin metathesis, catalysis.

$\mathrm{N}$-heterocyclic carbenes (NHCs) have found widespread applications as important auxiliary ligands in modern organometallic and coordination chemistry [1-7]. Their unique steric and electronic properties have made possible the development of effective metal catalysts for various applications with the most outstanding examples in the field of ruthenium-catalyzed olefin metathesis [8-19]. Ruthenium complexes bearing NHC ligands usually demonstrate their superiority over first generation catalysts containing classical phosphine ligands exhibiting higher thermal stability, activity and selectivity (Figure 1) [4,20-23].
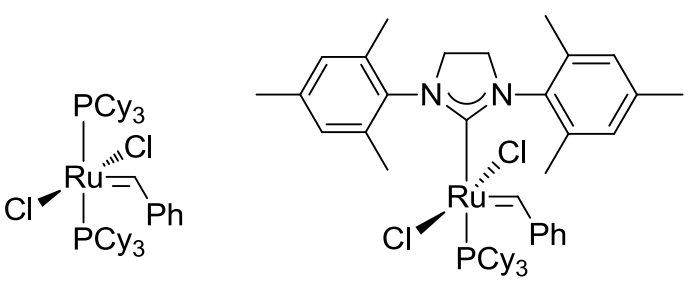

G-II

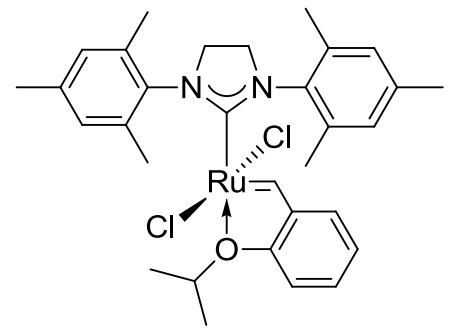

H-II

Figure 1. Commercially available olefin metathesis catalysts

A major advantage of this class of complexes is a possibility to perform a fine-tuning of their catalytic properties by modifying the NHC stereoelectronics [24-28]. In this context, the 
importance of unsymmetrical N-heterocyclic carbenes (uNHCs) as ligands in metal catalysis is doubtless, as desymmetrization allows for further fine-tuning. The introduction of functionality, chelation, chirality, and shielding effects can influence the catalyst stability, reactivity, and selectivity, thus motivating the exploration of new tailor-made systems [29].

On the other hand, fluorinated compounds have found extensive applications in pharmaceutical and medicinal chemistry [30-35] or crops [36,37] and material sciences [38,39], due to the unique physicochemical features of fluorine atoms introduced in organic molecules [40]. In the field of ruthenium-alkylidene complexes, the influence of fluorine and fluorinated groups on their catalytic properties has been mainly studied by usage of definitely modified phosphine [41-44], benzylidene [45-56] ligands, as well as by the replacement of one or two chlorine atoms at ruthenium, for example, with perfluoro-carboxylates [57-61] and -alkoxylates [62,63]. Meanwhile, the number of reports on metathesis catalysts decorated with fluorinated NHC ligands is extremely limited [64-67]. For instance, Fürstner et al. described an unsymmetrical complex with $\mathrm{C}_{6} \mathrm{~F}_{13}\left(\mathrm{CH}_{2}\right)_{2}$ group at one of the imidazolyl nitrogen to increase the solubility of the catalyst in supercritical $\mathrm{CO}_{2}$ [68]. In 2006, Grubbs reported rate acceleration in RCM of diethyl diallylmalonate arising from a $\mathrm{Ru} \cdots \mathrm{F}$ interaction between one ring of a $N, N^{\prime}$-bis(2,6difluorophenyl)imidazol-2-ylidene and the metal center in the Grubbs II catalyst analogue [69,70]. This work has been further extended with studies of synthesis and catalytic activities of closely related non-symmetrical analogues [71,72]. Consequently, the development of new tailor-made NHCs, particularly fluorinated ones, is highly desirable to enable new transformations or to bring established reactions into new reaction media.

At the same time, the variation of the substitution pattern in $\mathrm{N}$-aryl moieties of the NHC ligand led to extremely active catalysts for challenging transformations involving metathesis of hindered substrates [4,73-76]. However, it is not always easy to predict how structural and electronic changes at the NHC can affect the catalyst activity. For example, the presence of bulky substituents on nitrogen has been shown to improve catalyst stability limiting decomposition pathways due to $\mathrm{C}-\mathrm{H}$ bond activation of $\mathrm{N}$-aryl rings [77], whereas mono-ortho-substituted $\mathrm{N}$-aryl groups of the NHC ligand have been recognized as an important feature for successful ring-closing metathesis (RCM) reactions [78]. On the other hand, $N$-para-methoxyaryl NHCs [79] have been found to be excellent ligands for palladium catalysts for Buchwald-Hartwig coupling, compared to the corresponding parent NHCs [80,81]. Plenio et. al. have recently established that parafunctionalized $N$-aryl NHC complexes did indeed affect the properties of the transition metal centre [82]. Later Nolan et. al. have demonstrated that $\mathrm{Ru}$ metathesis catalysts bearing imidazoline-based NHCs with $N$-para-methoxyaryl moieties were able to increase the initiation rate in RCM reactions at very low catalyst loadings [83]. 
Based on our experience in metathesis of different unsaturated fluorine-containing molecules [84-92], we have recently developed an efficient route to novel type of metathesis catalysts comprising unsymmetrical imidazolinylidene ligand with hexafluoroisopropylmethoxy $\left[\left(\mathrm{CF}_{3}\right)_{2}(\mathrm{OMe}) \mathrm{C}-\right]$ group in one of the $N$-aryl substituents and have demonstrated their good performance in ring closing olefin metathesis [93] (1 and 2, Fig. 2). With the aim of evaluating the effects of mono-ortho-arylsubstitution and the replacement of para-methyl $\mathrm{N}$-aryl group with more electron-donating methoxy group in unsymmetrical fluorinated NHC ligand on the activity of the resulting precatalysts, we now want to disclose the synthesis of the corresponding ruthenium carbene complexes (3-6, Fig. 2) and preliminary evaluation of their catalytic activity as well.

\section{Previous work:}
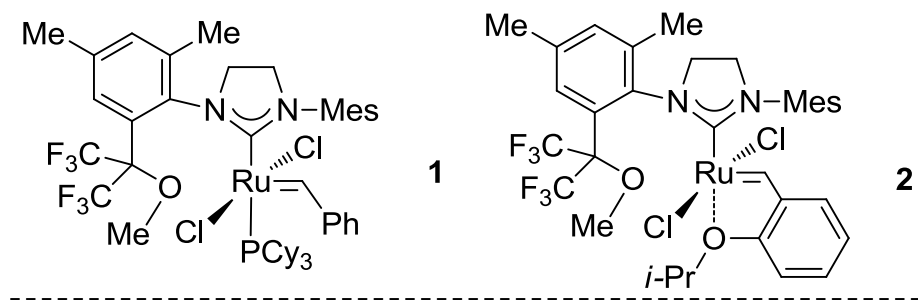

This work:
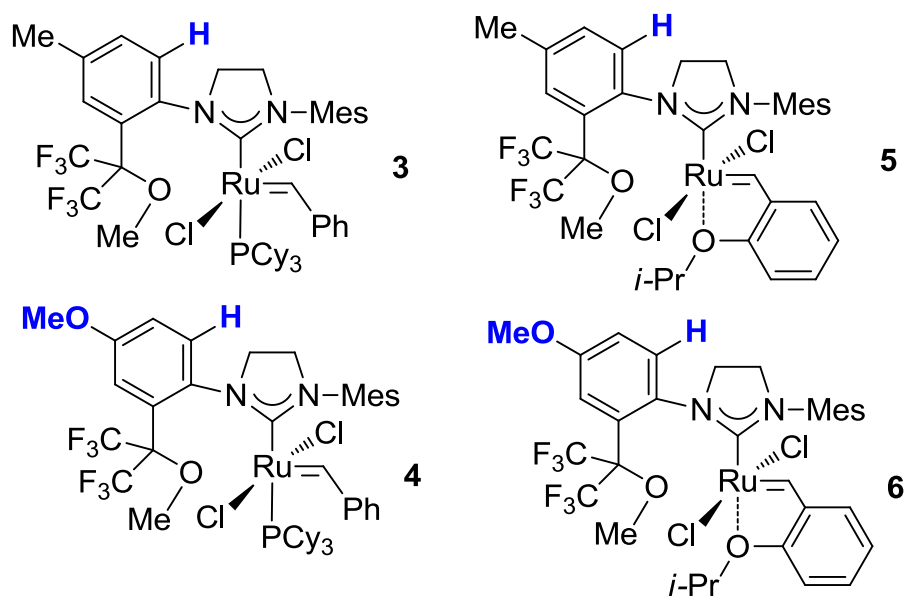

Figure 2

\section{Results and discussion}

First, we directed our efforts towards the synthesis of the mono-substituted anilines 10a,b containing hexafluoroisopropylmethoxy group in the ortho-position. For this purpose the commercially available para-toluidine and para-anisidine were heated with excess of hexafluoroacetone (HFA) hydrate under acid catalysis according to previously elaborated for 2,4dimethyl derivative protocol [93] to give the corresponding anilines $\mathbf{7 a , b}$ in good to excellent yields. To perform the selective $O$-methylation, they were first transformed into azides $\mathbf{8 a}, \mathbf{b}$ via successive treatments with sodium nitrite and azide, and then, $\mathbf{8 a , b}$ were methylated with methyl iodide under basic conditions to afford $O$-protected azides $\mathbf{9 a , b}$. Finally, the reduction of azido group of 9 was performed with sodium borohydride under cobalt dichloride-catalysis in the 
presence of cetyltrimethylammonium bromide to stabilize catalytic Co-species leading to excellent yields of anilines 10a,b (Scheme 1).
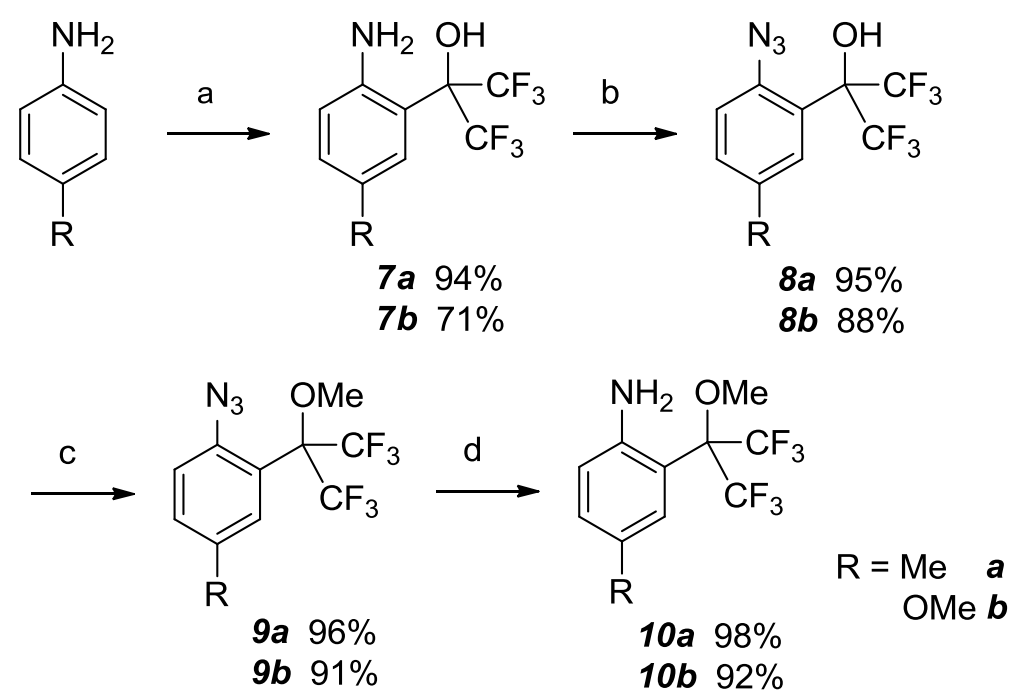

(a) HFA $1.5 \mathrm{H}_{2} \mathrm{O}$, pTSA, $100{ }^{\circ} \mathrm{C}, 8 \mathrm{~h}$, (b) $\mathrm{NaNO}_{2}, \mathrm{H}_{2} \mathrm{SO}_{4}, 0^{\circ} \mathrm{C}, \mathrm{NaN}_{3}, \mathrm{H}_{2} \mathrm{O}, \mathrm{rt}, 1 \mathrm{~h}$, (c) $\mathrm{MeI}, \mathrm{K}_{2} \mathrm{CO}_{3}$, MeCN, rt, 2 days, (d) $\mathrm{NaBH}_{4}, \mathrm{CoCl}_{2}, \mathrm{CTABr}, \mathrm{H}_{2} \mathrm{O} / \mathrm{MeOH}, \mathrm{rt}, 1$ h.

\section{Scheme 1}

The anilines 10a,b obtained were further used as the starting materials to construct the desired imidazolinium salts 16a,b. The synthetic sequence included: (a,b) selective $N$-acylation with chloroacetyl chloride with subsequent halogen exchange with $\mathrm{NaI}$ to give the iodides $\mathbf{1 2 a}$,b in very good yields for both steps; (c,d) condensation with mesitylamine followed by the reduction of the resulting aminoamides $\mathbf{1 3 a}, \mathbf{b}$ with $\mathrm{BH}_{3} \cdot \mathrm{SMe}_{2}$ to afford the corresponding diamines $\mathbf{1 4 a}, \mathbf{b}$; and finally $(e, f)$ selective formylation at the more sterically accessible amino group using acetic-formic mixed anhydride followed by heterocyclization via the treatment with stoichiometric amounts of triflic acid $(\mathrm{TfOH})$ and triflic anhydride $\left(\mathrm{Tf}_{2} \mathrm{O}\right)$ to give the target $\mathrm{NHC}$ precursors $\mathbf{1 6 \mathbf { a }}$ and $\mathbf{1 6} \mathbf{b}$ in $85 \%$ and $83 \%$ yields, respectively (Scheme 2). The last two steps (e,f) were performed using a slightly modified procedure previously developed by us for sterically demanding fluorinated imidazolinium salts [93]. 

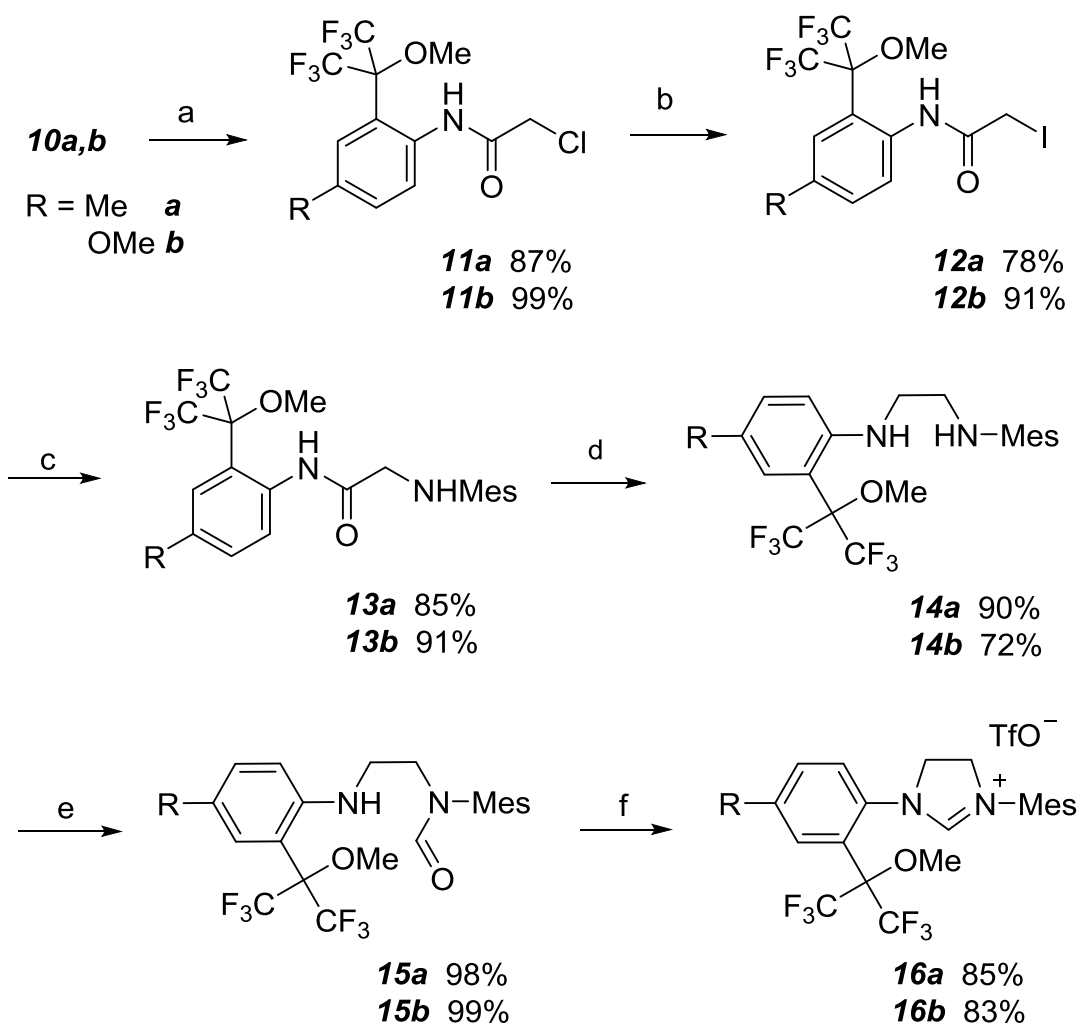

(a) $\mathrm{ClCOCH}_{2} \mathrm{Cl}, \mathrm{AcOH}, \mathrm{AcONa}, \mathrm{H}_{2} \mathrm{O}$, rt, $10 \mathrm{~min}$, (b) NaI, acetone, rt, 1 day, (c) $\mathrm{MesNH}_{2}, \mathrm{rt}, 4$ days, (d) $\mathrm{BH}_{3} \cdot \mathrm{SMe}_{2}, \mathrm{PhMe}, 90{ }^{\circ} \mathrm{C}, 4 \mathrm{~h}$, (e) AcOCHO, DCM, rt, 5 min., (f) 1. TfOH, rt, 2. $\mathrm{Tf}_{2} \mathrm{O}$, $65^{\circ} \mathrm{C}, 3$. DIPEA, $80{ }^{\circ} \mathrm{C}$, PhMe, $3 \mathrm{~h}$.

Scheme 2

With these new fluorinated unsymmetrical NHC salts in hand, we prepared the new ruthenium complexes 3-6 in moderate to good yields following the conventional route including the reactions of in situ generated carbene with commercially available $\mathrm{RuCl}_{2}\left(\mathrm{PCy}_{3}\right)_{2}(=\mathrm{CHPh}) \mathbf{G}-\mathbf{I}$ [94] and $\mathrm{RuCl}_{2}\left(\mathrm{PCy}_{3}\right)\left(=\mathrm{CH}\left({ }^{-}{ }^{i}{ }^{P r O}-\mathrm{C}_{6} \mathrm{H}_{4}\right)\right) \mathbf{H}-\mathbf{I}$ [95]. Purification by silica gel chromatography and further recrystallization from a DCM/n-pentane mixture afforded dark-brown $(\mathbf{3}, \mathbf{4})$ and dark-green $(\mathbf{5}, \mathbf{6})$ air stable solids (Scheme 3).

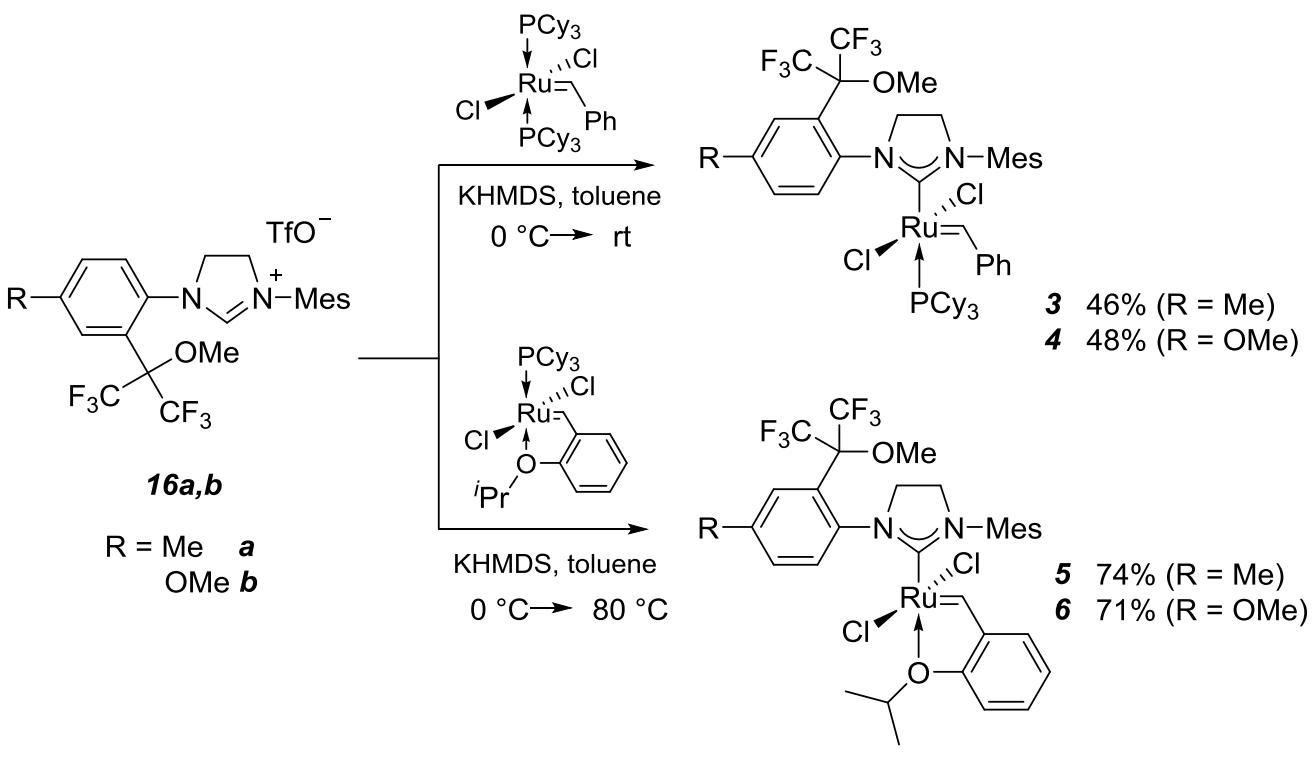

Scheme 3 
The complexes obtained were fully characterized by NMR spectroscopy and elemental analysis. In ${ }^{1} \mathrm{H}$ NMR spectra of both phosphine-containing 3, 4 and phosphine-free 5, 6 complexes measured at room temperature the absorptions of intrinsic benzylidene protons are observed around $19.6 \mathrm{ppm}$ and $16.9 \mathrm{ppm}$, respectively, as two broad singlets in each case. The doubling of the signals for $\mathrm{CF}_{3}$-groups takes place in ${ }^{19} \mathrm{~F}$ NMR spectra also. This phenomenon could be attributed to the existence of two rotamers due to hindered rotation of bulky fluorinated aryl group around $\mathrm{C}-\mathrm{N}$ bond. These doublets come together at $60-70{ }^{\circ} \mathrm{C}$ under NMR experiment measured in toluene- $d 8$, which supports the existence of rotamers (see Experimental section). The ${ }^{13} \mathrm{C}$ NMR spectrum of the $\mathrm{NHC}$ at $60^{\circ} \mathrm{C}$ displays a resonance at $220 \mathrm{ppm}$ for the carbene center, which is in the expected range for aryl-substituted imidazolylidenes (see Experimental section). In addition, single crystals of good quality for X-ray analysis from complexes 5 and 6 (Fig. 3) were obtained by slow diffusion of pentane into a concentrated dichloromethane solution.

<InlineShape1>
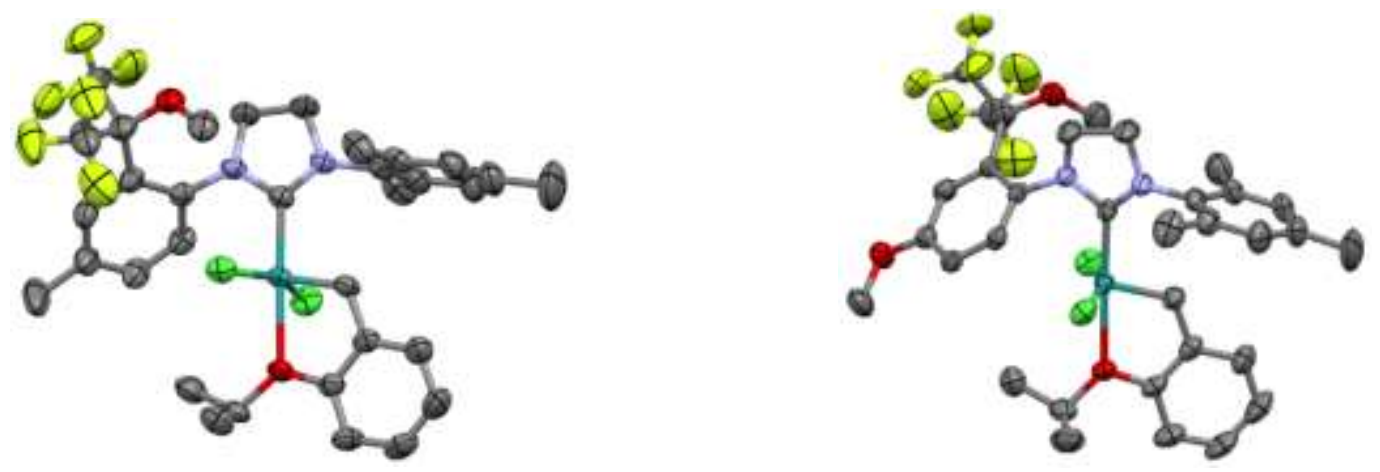

5

6

Figure 3. Molecular structure of complexes $\mathbf{5}$ and 6. Thermal ellipsoids are drawn at $30 \%$ probability. Hydrogen atoms are omitted for clarity.

Catalytic activities of the prepared catalysts 3-6 were investigated in RCM reactions with diethyl diallylmalonate (DEDAM) and in CM reaction of allylbenzene with 1,4-diacetoxybut-2ene following standard protocols for evaluation of olefin metathesis catalysts [71,72]. The commercially available G-II and H-II catalysts along with previously obtained $\mathbf{1}$ and $\mathbf{2}$ were used as reference catalysts to find out how modulating the electronic and steric changes of the fluoroakyl-substituted aryl ring might affect the catalytic activity.

As a result, we found that initiation rates of the Grubbs type catalyst 3,4 in RCM of DEDAM were slightly higher as compared to G-II and 1 (Figure 4). On the other hand, the initiation rates of Hoveyda type catalysts 5 and $\mathbf{6}$ have proved to be in between the corresponding $\mathbf{H}-\mathbf{I I}$ and $\mathbf{2}$ rates (Figure 5), exhibiting some significant initiation period (about $10 \mathrm{~min}$ ) before they could achieve full conversion in longer reaction times $(4 \mathrm{~h})$. 


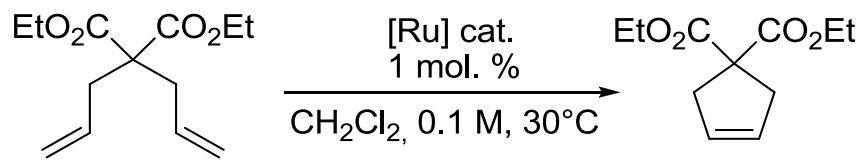

In the cross metathesis of allylbenzene with an excess of 1,3-diacetoxybut-2-ene, the phosphine-containing catalysts $\mathbf{3}$ and $\mathbf{4}$ revealed slightly higher reactivity as compared with the ortho,ortho-disubstituted analogue $\mathbf{1}$ and close kinetic profiles to G-I catalyst (Figure 6). In the case of phosphine-free complexes $\mathbf{5}$ and $\mathbf{6}$ these differences in catalytic activity did not exist and the complexes allowed to reach an equilibrium at 70-80\% conversion within 30 min (Figure 7).

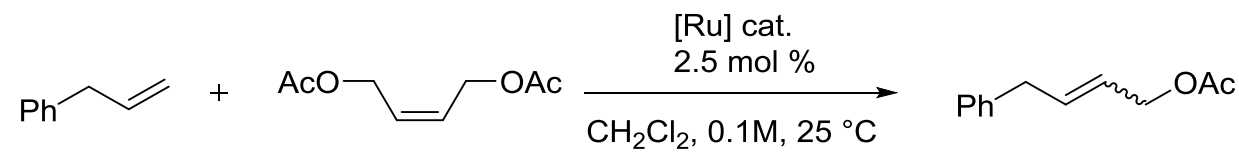

as compared to $\mathbf{1}, \mathbf{G}-\mathbf{I I}$ and $\mathbf{H}-\mathbf{I I}$

as compared to $\mathbf{2}, \mathbf{G}-\mathbf{I I}$ and $\mathbf{H}-\mathbf{I I}$

In all studied reaction, no significant influence of para-methoxyaryl substitution on the catalytic activity of complexes could be found.

\section{Conclusions}

In conclusion, the synthesis of new metathesis catalysts bearing unsymmetrical fluorinated NHC ligands with hexafluoroisopropylmethoxy group in ortho-position of $\mathrm{N}$-aryl-substituent have been developed. The effects of mono-ortho-arylsubstitution and the replacement of para-methyl $\mathrm{N}$-aryl group with more electron-donating methoxy group in unsymmetrical fluorinated NHC ligand on the activity of complexes have been evaluated. As a result, the performance of the new Grubbs type catalysts in olefin metathesis has proved to be similar to the classical Grubbs second generation catalyst. On the other hand, the new Hoveyda type catalysts demonstrate a short latent character before reaching full conversion in RCM of diallylmalonate in longer period in comparison to the commercially available Hoveyda-II catalyst equipped with symmetrical $\mathrm{H}_{2} \mathrm{IMes}$ carbene ligand. In all cases, the absence of a methyl group at the other ortho-position of the hexafluoroisopropylmethoxy ortho-substituted aryl group of the unsymmetrical NHC led to faster initial rates with Grubbs type catalyst $(\mathbf{3}, \mathbf{4}$ as compared to $\mathbf{1})$ and with Hoveyda type catalysts after the initiation period was completed (5, $\mathbf{6}$ as compared to $\mathbf{2})$.

\section{Acknowledgments}

This work was financially supported by the Russian Scientific Foundation (grant RSF № 16-1310364). S.M. thanks the RAS and the French Embassy in Moscow for a Ph.D.

\section{Experimental}

\subsection{General remarks.}

All solvents were freshly distilled from appropriate drying agents before use. All other reagents were recrystallized or distilled as necessary. Syntheses of ruthenium complexes were performed under an argon atmosphere using a standard Schlenk technique. Analytical TLC was performed 
with Merck silica gel 60 F254 plates. Visualization was accomplished by UV light (254 and $366 \mathrm{~nm})$, spraying by $\mathrm{Ce}\left(\mathrm{SO}_{4}\right)_{2}$ solution in $5 \% \mathrm{H}_{2} \mathrm{SO}_{4}$ or $\mathrm{KMnO}_{4}$ solution in water. Column chromatography was carried out using Merck silica gel 60 (230-400 mesh ASTM) and ethyl acetate/petroleum ether. NMR spectra were recorded, unless otherwise stated, at room temperature on Bruker AV-300, AV-400, AV-500, AV-600 spectrometers operating at 300, 400, 500, and 600 $\mathrm{MHz}$ for ${ }^{1} \mathrm{H} ; 75,101,126$, and $151 \mathrm{MHz}$ for ${ }^{13} \mathrm{C} ; 282,376,471$, and $564 \mathrm{MHz}$ for ${ }^{19} \mathrm{~F}\left(\mathrm{CFCl}_{3}\right.$ as reference), and $121,162,202$, and $243 \mathrm{MHz}$ for ${ }^{31} \mathrm{P}\left(85 \% \mathrm{H}_{3} \mathrm{PO}_{4}\right.$ as reference $)$. The chemical shifts are frequency referenced relative to the residual undeuterated solvent peaks.

\subsection{General procedure for synthesis of 7.}

A mixture of 6-methylaniline or 6-methoxyaniline (46.7 mmol), hexafluoroacetone sesquihydrate (18.6 g, $112.1 \mathrm{mmol})$, and PTSA (100 mg, $0.5 \mathrm{mmol})$ was heated at $100^{\circ} \mathrm{C}$ for $20 \mathrm{~h}$. After cooling to r.t., water $(50 \mathrm{~mL})$ was added and a resulting mixture was extracted with EtOAc $(3 \times 30 \mathrm{~mL})$. The combined organic layers were washed with $\mathrm{H}_{2} \mathrm{O}$ and brine and then dried over $\mathrm{MgSO}_{4}$. The solvent was removed under reduced pressure, and the resulting solid was recrystallized from petroleum ether to yield a dark solid.

\subsubsection{2-(2-Amino-5-methylphenyl)-1,1,1,3,3,3-hexafluoropropan-2-ol (7a).}

Yield: 94\%; brown solid; mp 110-113 ${ }^{\circ} \mathrm{C} ;{ }^{1} \mathrm{H}$ NMR $\left(300 \mathrm{MHz}, \mathrm{CDCl}_{3}\right) \delta 7.37$ (s, 1H), $7.16(\mathrm{~d}$, $\left.{ }^{3} J_{\mathrm{H}, \mathrm{H}}=8.0 \mathrm{~Hz}, 1 \mathrm{H}\right), 6.97\left(\mathrm{~d},{ }^{3} J_{\mathrm{H}, \mathrm{H}}=8.0 \mathrm{~Hz}, 1 \mathrm{H}\right), 5.57$ (br.s, 3H), 2.35 (s, 3H); ${ }^{13} \mathrm{C} \mathrm{NMR}(75 \mathrm{MHz}$, $\left.\mathrm{CDCl}_{3}\right) \delta 138.8,135.3,131.3,129.1,127.4,123.5\left(\mathrm{q},{ }^{1} J_{\mathrm{C}, \mathrm{F}}=288 \mathrm{~Hz}\right), 123.0,80.0\left(\mathrm{p},{ }^{2} J_{\mathrm{C}, \mathrm{F}}=30\right.$ $\mathrm{Hz}), 21.2 ;{ }^{19} \mathrm{~F}$ NMR $\left(282 \mathrm{MHz}, \mathrm{CDCl}_{3}\right) \delta-75.14$ (s). Anal. Calcd for $\mathrm{C}_{10} \mathrm{H}_{9} \mathrm{~F}_{6} \mathrm{NO}(\%) \mathrm{C}, 43.97 ; \mathrm{H}$, 3.32; N, 5.13. Found: C, 43.92; H, 3.38; N, 4.90.

\subsubsection{2-(2-Amino-5-methoxyphenyl)-1,1,1,3,3,3-hexafluoropropan-2-ol (7b).}

Yield: 71\%; black solid; mp 104-107 ${ }^{\circ} \mathrm{C} ;{ }^{1} \mathrm{H}$ NMR (400 MHz, $\left.\mathrm{CDCl}_{3}\right) \delta 7.13(\mathrm{~s}, 1 \mathrm{H}), 7.04\left(\mathrm{~d},{ }^{3} J_{\mathrm{H}, \mathrm{H}}\right.$ $=8.7 \mathrm{~Hz}, 1 \mathrm{H}), 6.90\left(\mathrm{dd}, J_{\mathrm{H}, \mathrm{H}}=8.7,2.7 \mathrm{~Hz}, 1 \mathrm{H}\right), 6.08$ (br.s, $\left.3 \mathrm{H}\right), 3.79(\mathrm{~s}, 3 \mathrm{H}) ;{ }^{13} \mathrm{C} \mathrm{NMR}(101 \mathrm{MHz}$, $\left.\mathrm{CDCl}_{3}\right) \delta 157.3,133.6,129.4,125.0,123.4\left(\mathrm{q},{ }^{1} \mathrm{~J}_{\mathrm{C}, \mathrm{F}}=289 \mathrm{~Hz}\right), 115.8,114.5-114.3(\mathrm{~m}), 81.0-$ 79.0 (m), 55.7; ${ }^{19} \mathrm{~F}$ NMR (376 MHz, $\left.\mathrm{CDCl}_{3}\right) \delta-75.33$ (s). Anal. Calcd for $\mathrm{C}_{10} \mathrm{H}_{9} \mathrm{~F}_{6} \mathrm{NO}_{2}(\%) \mathrm{C}$, 41.53; H, 3.14; N, 4.84. Found: C, 41.57; H, 3.37; N, 4.65.

\subsection{General procedure for synthesis of 8 .}

To a mixture of aniline $7(20 \mathrm{mmol})$ in $60 \mathrm{ml}$ of water concentrated sulfuric acid $(10.7 \mathrm{~mL}$, $200 \mathrm{mmol}$ ) was slowly added. The resulting mixture was cooled to $0^{\circ} \mathrm{C}$ and solution of $\mathrm{NaNO}_{2}$ $(1.59 \mathrm{~g}, 23.0 \mathrm{mmol})$ in $6 \mathrm{~mL}$ of water was added dropwise and reaction mixture was stirred in an ice bath for $30 \mathrm{~min}$. Then solution of $\mathrm{NaN}_{3}(1.56 \mathrm{~g}, 24 \mathrm{mmol})$ in $6 \mathrm{~mL}$ of water was added dropwise. After full addition the reaction mixture was allowed to stir at r.t. for 3 hours. Then the reaction mixture was extracted with EtOAc $(3 \times 20 \mathrm{~mL})$. Combined organic layer was washed with brine $(20 \mathrm{~mL})$, dried over $\mathrm{MgSO}_{4}$ and concentrated under reduced pressure. The crude product was 
purified by flash chromatography using EtOAc/petroleum ether (1:3) as eluent to yield yellowish crystals.

4.3.1. 2-(2-Azido-5-methylphenyl)-1,1,1,3,3,3-hexafluoropropan-2-ol (8a).

Yield: 95\%; mp 64-65 ${ }^{\circ}$; ${ }^{1} \mathrm{H}$ NMR (400 MHz, $\left.\mathrm{CDCl}_{3}\right) \delta 7.44(\mathrm{~s}, 1 \mathrm{H}), 7.33\left(\mathrm{~d},{ }^{3} \mathrm{~J}_{\mathrm{H}, \mathrm{H}}=8.2 \mathrm{~Hz}, 1 \mathrm{H}\right)$, $7.16\left(\mathrm{~d},{ }^{3} J_{\mathrm{H}, \mathrm{H}}=8.3 \mathrm{~Hz}, 1 \mathrm{H}\right), 7.04$ (br.s, $\left.1 \mathrm{H}\right), 2.38$ (s, 3H); ${ }^{13} \mathrm{C} \mathrm{NMR}\left(101 \mathrm{MHz}, \mathrm{CDCl}_{3}\right) \delta 135.9$, 135.4, 132.3, $130.3(\mathrm{~m}), 122.7\left(\mathrm{q},{ }^{1} J_{\mathrm{C}, \mathrm{F}}=289 \mathrm{~Hz}\right), 119.8,119.7,79.9\left(\mathrm{p},{ }^{2} J_{\mathrm{C}, \mathrm{F}}=30 \mathrm{~Hz}\right), 21.2 ;{ }^{19} \mathrm{~F}$ NMR (376 MHz, $\left.\mathrm{CDCl}_{3}\right) \delta-74.88$ (s). Anal. Calcd for $\mathrm{C}_{10} \mathrm{H}_{7} \mathrm{~F}_{6} \mathrm{~N}_{3} \mathrm{O}(\%) \mathrm{C}, 40.15 ; \mathrm{H}, 2.36 ; \mathrm{N}$, 14.05. Found: C, 39.91; H, 2.39; N, 14.19.

4.3.2. 2-(2-Azido-5-methoxyphenyl)-1,1,1,3,3,3-hexafluoropropan-2-ol ( $8 \boldsymbol{b})$.

Yield: 88\%; mp 67-68 ${ }^{\circ} \mathrm{C}$; ${ }^{1} \mathrm{H}$ NMR (400 MHz, $\left.\mathrm{CDCl}_{3}\right) \delta 7.19$ (d, $\left.J=8.8 \mathrm{~Hz}, 2 \mathrm{H}\right), 7.19$ (s, 1H), 7.07 (dd, $J=8.9,2.7 \mathrm{~Hz}, 1 \mathrm{H}), 7.07$ (br.s, $1 \mathrm{H}$ ), 3.82 (s, 3H); ${ }^{13} \mathrm{C} \mathrm{NMR}\left(101 \mathrm{MHz}, \mathrm{CDCl}_{3}\right) \delta 157.1$, $130.3,122.7\left(\mathrm{~d},{ }^{1} J_{\mathrm{C}, \mathrm{F}}=289 \mathrm{~Hz}\right), 121.0,120.8,116.9,115.8\left(\mathrm{p},{ }^{3} J_{\mathrm{C}, \mathrm{F}}=3 \mathrm{~Hz}\right), 79.8\left(\mathrm{p},{ }^{2} J_{\mathrm{C}, \mathrm{F}}=30\right.$ $\mathrm{Hz}$ ), 55.8; ${ }^{19} \mathrm{~F}$ NMR (376 MHz, $\left.\mathrm{CDCl}_{3}\right) \delta-74.94$ (s). Anal. Calcd for $\mathrm{C}_{10} \mathrm{H}_{7} \mathrm{~F}_{6} \mathrm{~N}_{3} \mathrm{O}_{2}$ (\%) C, 38.11; H, 2.24; N, 13.33. Found: C, 37.79; H, 2.32; N, 13.45 .

\subsection{General procedure for synthesis of 9.}

A mixture of azide $8(17.6 \mathrm{mmol})$, iodomethane $(2.2 \mathrm{~mL}, 35.3 \mathrm{mmol})$ and anhydrous $\mathrm{K}_{2} \mathrm{CO}_{3}(4.88$ $\mathrm{g}, 35.3 \mathrm{mmol}$ ) in $80 \mathrm{~mL}$ of acetonitrile was stirred at r.t. for 2 days. After reaction completion the solvent was evaporated under reduced pressure, residual solid was dispersed in EtOAc and filtered. The resulting filtrate was evaporated again and purified by flash chromatography using EtOAc/petroleum ether (1:8) as eluent to yield yellow oil that was crystallized after complete removal of solvent under high vacuum.

4.4.1. 1-Azido-2-(1,1,1,3,3,3-hexafluoro-2-methoxypropan-2-yl)-4-methylbenzene (9a).

Yield: 96\%; mp 51-52 ${ }^{\circ} \mathrm{C} ;{ }^{1} \mathrm{H}$ NMR (400 MHz, $\left.\mathrm{CDCl}_{3}\right) \delta 7.36(\mathrm{~s}, 1 \mathrm{H}), 7.33\left(\mathrm{~d},{ }^{3} J_{\mathrm{H}, \mathrm{H}}=8.3 \mathrm{~Hz}, 1 \mathrm{H}\right)$, $7.21\left(\mathrm{~d},{ }^{3} J_{\mathrm{H}, \mathrm{H}}=8.2 \mathrm{~Hz}, 1 \mathrm{H}\right), 3.46(\mathrm{~s}, 3 \mathrm{H}), 2.38(\mathrm{~s}, 3 \mathrm{H}) ;{ }^{13} \mathrm{C} \mathrm{NMR}\left(101 \mathrm{MHz}, \mathrm{CDCl}_{3}\right) \delta 137.2$, 135.0, 132.6, 131.8, $122.6\left(\mathrm{q},{ }^{1} J_{\mathrm{C}, \mathrm{F}}=292 \mathrm{~Hz}\right), 120.7,118.2,84.3\left(\mathrm{p},{ }^{2} J_{\mathrm{C}, \mathrm{F}}=29 \mathrm{~Hz}\right), 54.6,21.1 ;{ }^{19} \mathrm{~F}$ NMR (376 MHz, $\left.\mathrm{CDCl}_{3}\right) \delta-69.86$ (s). Anal. Calcd for $\mathrm{C}_{11} \mathrm{H}_{9} \mathrm{~F}_{6} \mathrm{~N}_{3} \mathrm{O}(\%) \mathrm{C}, 42.18 ; \mathrm{H}, 2.90 ; \mathrm{N}$, 13.42. Found: C, 42.35; H, 3.11; N, 13.72.

4.4.2. 1-Azido-2-(1,1,1,3,3,3-hexafluoro-2-methoxypropan-2-yl)-4-methoxybenzene (9b).

Yield: 91\%; mp 52-53 ${ }^{\circ} \mathrm{C} ;{ }^{1} \mathrm{H}$ NMR (400 MHz, $\left.\mathrm{CDCl}_{3}\right) \delta 7.25\left(\mathrm{~d},{ }^{3} J_{\mathrm{H}, \mathrm{H}}=8.8 \mathrm{~Hz}, 1 \mathrm{H}\right), 7.13(\mathrm{~s}, 1 \mathrm{H})$, $7.07(\mathrm{dd}, J=8.8,2.8 \mathrm{~Hz}, 1 \mathrm{H}), 3.83(\mathrm{~s}, 3 \mathrm{H}), 3.48(\mathrm{~s}, 3 \mathrm{H}) ;{ }^{13} \mathrm{C} \mathrm{NMR}\left(101 \mathrm{MHz}, \mathrm{CDCl}_{3}\right) \delta 156.8$, $132.2,122.5\left(\mathrm{q},{ }^{1} J_{\mathrm{C}, \mathrm{F}}=291 \mathrm{~Hz}\right), 122.0,119.6,117.5-117.3(\mathrm{~m}), 117.1,84.9-83.4(\mathrm{~m}), 55.8,54.7$; ${ }^{19} \mathrm{~F}$ NMR (376 MHz, $\left.\mathrm{CDCl}_{3}\right) \delta-69.87$ (s). Anal. Calcd for $\mathrm{C}_{11} \mathrm{H}_{9} \mathrm{~F}_{6} \mathrm{~N}_{3} \mathrm{O}_{2}(\%) \mathrm{C}, 40.13 ; \mathrm{H}, 2.76 ; \mathrm{N}$, 12.76. Found: C, 40.41; H, 2.83; N, 12.97.

4.5. General procedure for synthesis of $\mathbf{1 0}$. 
The azide $9(13.8 \mathrm{mmol})$ was dissolved in $4.3 \mathrm{ml}$ of methanol, then $\mathrm{CoCl}_{2} \cdot 6 \mathrm{H}_{2} \mathrm{O}(290 \mathrm{mg}, 1.4$ mmol) and cetyltrimethylammonium bromide (CTABr) (426 mg, $1.4 \mathrm{mmol}$ ) were added with stirring at r.t. Then a solution of $\mathrm{NaBH}_{4}(1.04 \mathrm{~g}, 27.6 \mathrm{mmol})$ in $26 \mathrm{ml}$ of water was added dropwise. After full addition the reaction mixture was stirred another $30 \mathrm{~min}$ and extracted with $\mathrm{Et}_{2} \mathrm{O}(3 \times 15$ $\mathrm{ml}$ ). Combined organic layer was washed with $10 \mathrm{ml}$ of water, filtered through cotton wool and evaporated under reduced pressure to yield yellow crystals.

4.5.1. 2-(1,1,1,3,3,3-Hexafluoro-2-methoxypropan-2-yl)-4-methylaniline (10a).

Yield: 98\%; mp 61-63 ${ }^{\circ}$; ${ }^{1} \mathrm{H}$ NMR $\left(400 \mathrm{MHz}, \mathrm{CDCl}_{3}\right) \delta 7.09(\mathrm{~s}, 1 \mathrm{H}), 7.06\left(\mathrm{~d},{ }^{3} \mathrm{~J}_{\mathrm{H}, \mathrm{H}}=8.3 \mathrm{~Hz}, 1 \mathrm{H}\right)$, $6.65\left(\mathrm{~d},{ }^{3} J_{\mathrm{H}, \mathrm{H}}=8.2 \mathrm{~Hz}, 1 \mathrm{H}\right), 4.56$ (br.s, $\left.2 \mathrm{H}\right), 3.56$ (s, 3H), $2.26(\mathrm{~s}, 3 \mathrm{H}) ;{ }^{13} \mathrm{C} \mathrm{NMR}(101 \mathrm{MHz}$, $\left.\mathrm{CDCl}_{3}\right) \delta 144.1,132.1,130.1\left(\mathrm{p},{ }^{3} J_{\mathrm{C}, \mathrm{F}}=2.1 \mathrm{~Hz}\right), 127.5,123.0\left(\mathrm{q},{ }^{1} J_{\mathrm{C}, \mathrm{F}}=292 \mathrm{~Hz}\right), 118.9,109.4$, $85.3\left(\mathrm{p},{ }^{2} J_{\mathrm{C}, \mathrm{F}}=28 \mathrm{~Hz}\right), 54.8,20.7 ;{ }^{19} \mathrm{~F} \mathrm{NMR}\left(376 \mathrm{MHz}, \mathrm{CDCl}_{3}\right) \delta-69.84$ (s). Anal. Calcd for $\mathrm{C}_{11} \mathrm{H}_{11} \mathrm{~F}_{6} \mathrm{NO}(\%) \mathrm{C}, 46.00 ; \mathrm{H}, 3.86 ; \mathrm{N}, 4.88$. Found: C, 45.92; H, 3.97; N, 5.03.

4.5.2. 2-(1,1,1,3,3,3-Hexafluoro-2-methoxypropan-2-yl)-4-methoxyaniline (10b).

Yield: $92 \%$; mp 51-53 ${ }^{\circ} \mathrm{C} ;{ }^{1} \mathrm{H}$ NMR $\left(400 \mathrm{MHz}, \mathrm{CDCl}_{3}\right) \delta 6.88(\mathrm{~s}, 1 \mathrm{H}), 6.88\left(\mathrm{~d},{ }^{3} \mathrm{~J}_{\mathrm{H}, \mathrm{H}}=9.4 \mathrm{~Hz}, 1 \mathrm{H}\right)$, $6.69\left(\mathrm{~d},{ }^{3} J_{\mathrm{H}, \mathrm{H}}=9.4 \mathrm{~Hz}, 1 \mathrm{H}\right), 4.42$ (br.s, 2H), 3.75 (s, 3H), 3.57 (s, 3H); ${ }^{13} \mathrm{C}$ NMR $(101 \mathrm{MHz}$, $\left.\mathrm{CDCl}_{3}\right) \delta 152.1,140.4,123.0\left(\mathrm{q},{ }^{1} J_{\mathrm{C}, \mathrm{F}}=292 \mathrm{~Hz}\right), 120.0,118.0,115.3-115.1(\mathrm{~m}), 110.3,85.9-84.3$ (m), 55.9, 54.9; $\left.{ }^{19} \mathrm{~F} \mathrm{NMR} \mathrm{(376} \mathrm{MHz,} \mathrm{CDCl}_{3}\right) \delta-69.87$ (s). Anal. Calcd for $\mathrm{C}_{11} \mathrm{H}_{11} \mathrm{~F}_{6} \mathrm{NO}_{2}(\%) \mathrm{C}$, 43.57; H, 3.66; N, 4.62. Found: C, 43.89; H, 4.01; N, 4.64.

4.6. General procedure for synthesis of 11.

Aniline 10 (10.6 mmol) was added to $6 \mathrm{~mL}$ of glacial acetic acid in a round-bottomed flask followed by chloroacetyl chloride $(870 \mu \mathrm{L}, 10.9 \mathrm{mmol})$ and $10.4 \mathrm{~mL}$ of half-saturated aqueous sodium acetate. Precipitation of the amide was observed in $10 \mathrm{~min}$. The product is stirred thoroughly with $10 \mathrm{~mL}$ of cold water and isolated by filtration. Solid product was dissolved in ethyl acetate and washed with saturated aqueous $\mathrm{NaHCO}_{3}$ and brine, dried above $\mathrm{MgSO}_{4}$. Then solvent was removed under reduced pressure, resulting solid residue was recrystallized in petroleum ether to yield a white solid.

4.6.1. 2-Chloro-N-[2-(1,1,1,3,3,3-hexafluoro-2-methoxypropan-2-yl)-4-methylphenyl]acetamide (11a).

Yield: 87\%; $\mathrm{mp} 70-71{ }^{\circ} \mathrm{C} ;{ }^{1} \mathrm{H} \mathrm{NMR}\left(400 \mathrm{MHz}, \mathrm{CDCl}_{3}\right) \delta 9.78(\mathrm{~s}, 1 \mathrm{H}), 8.43\left(\mathrm{~d},{ }^{3} \mathrm{~J}_{\mathrm{H}, \mathrm{H}}=8.5 \mathrm{~Hz}, 1 \mathrm{H}\right)$, $7.32\left(\mathrm{~d},{ }^{3} J_{\mathrm{H}, \mathrm{H}}=8.6 \mathrm{~Hz}, 1 \mathrm{H}\right), 7.29(\mathrm{~s}, 1 \mathrm{H}), 4.19(\mathrm{~s}, 2 \mathrm{H}), 3.54(\mathrm{~s}, 3 \mathrm{H}), 2.37(\mathrm{~s}, 3 \mathrm{H}) ;{ }^{13} \mathrm{C}$ NMR $(101$ $\left.\mathrm{MHz}, \mathrm{CDCl}_{3}\right) \delta 164.0,134.9,134.8,132.2,130.2(\mathrm{~m}), 123.21(\mathrm{~s}), 122.5$ (q, $\left.{ }^{1} J_{\mathrm{C}, \mathrm{F}}=290 \mathrm{~Hz}\right), 114.8$, $85.1\left(\mathrm{p},{ }^{2} J_{\mathrm{C}, \mathrm{F}}=28.6 \mathrm{~Hz}\right), 54.7,43.2,21.2 ;{ }^{19} \mathrm{~F} \mathrm{NMR}\left(376 \mathrm{MHz}, \mathrm{CDCl}_{3}\right) \delta-69.57$ (s). Anal. Calcd for $\mathrm{C}_{13} \mathrm{H}_{12} \mathrm{ClF}_{6} \mathrm{NO}_{2}(\%) \mathrm{C}, 42.93 ; \mathrm{H}, 3.33 ; \mathrm{N}, 3.85$. Found: C, 43.11; H, 3.67; N, 4.12.

4.6.2.

2-Chloro-N-[2-(1,1,1,3,3,3-hexafluoro-2-methoxypropan-2-yl)-4methoxyphenyl]acetamide (11b). 
Yield: $99 \%$; mp 101-102 ${ }^{\circ} \mathrm{C} ;{ }^{1} \mathrm{H}$ NMR $\left(400 \mathrm{MHz}, \mathrm{CDCl}_{3}\right) \delta 9.62(\mathrm{~s}, 1 \mathrm{H}), 8.41\left(\mathrm{~d},{ }^{3} \mathrm{~J}_{\mathrm{H}, \mathrm{H}}=9.9 \mathrm{~Hz}\right.$, $1 \mathrm{H}), 7.05\left(\mathrm{~d},{ }^{3} \mathrm{~J}_{\mathrm{H}, \mathrm{H}}=9.9 \mathrm{~Hz}, 1 \mathrm{H}\right), 7.06(\mathrm{~s}, 1 \mathrm{H}), 4.19(\mathrm{~s}, 2 \mathrm{H}), 3.82(\mathrm{~s}, 3 \mathrm{H}), 3.54(\mathrm{~s}, 3 \mathrm{H}) ;{ }^{13} \mathrm{C} \mathrm{NMR}$ $\left(101 \mathrm{MHz} \mathrm{CDCl}_{3}\right) \delta 163.9,156.4,130.1,125.0,122.5\left(\mathrm{q},{ }^{1} J_{\mathrm{C}, \mathrm{F}}=289 \mathrm{~Hz}\right), 116.7,116.4-116.1$ $(\mathrm{m}), 115.9,84.9\left(\mathrm{p},{ }^{2} J_{\mathrm{C}, \mathrm{F}}=29 \mathrm{~Hz}\right), 55.7,54.8,43.1 ;{ }^{19} \mathrm{~F} \mathrm{NMR}\left(376 \mathrm{MHz}, \mathrm{CDCl}_{3}\right) \delta-69.63(\mathrm{~s})$. Anal. Calcd for $\mathrm{C}_{13} \mathrm{H}_{12} \mathrm{ClF}_{6} \mathrm{NO}_{3}(\%)$ C, 41.12; H, 3.19; N, 3.69. Found: C, 41.10; H, 3.22; N, 3.43. 4.7. General procedure for synthesis of $\mathbf{1 2 .}$

Chloroacetamide 11 (9.18 mmol) was dissolved in acetone $(90 \mathrm{~mL})$, and then sodium iodide ( $3.44 \mathrm{~g}, 23.0 \mathrm{mmol}$ ) was added. The reaction mixture was stirred at r.t. for $24 \mathrm{~h}$. After filtration, the mother liquor was concentrated, and the residue was dissolved in EtOAc $(20 \mathrm{~mL})$ and washed with a $5 \%$ solution of $\mathrm{Na}_{2} \mathrm{~S}_{2} \mathrm{O}_{3}(10 \mathrm{~mL})$. The organic layer was separated, and the aqueous layer was extracted with EtOAc $(3 \times 10 \mathrm{~mL})$. Combined organic layers were dried over $\mathrm{MgSO}_{4}$, filtered, and concentrated. The crude product was recrystallized from petroleum ether to yield a brown solid.

4.7.1. N-[2-(1,1,1,3,3,3-Hexafluoro-2-methoxypropan-2-yl)-4-methylphenyl]-2-iodoacetamide (12a).

Yield: 78\%; mp 98-99 ${ }^{\circ} \mathrm{C} ;{ }^{1} \mathrm{H}$ NMR $\left(400 \mathrm{MHz}, \mathrm{CDCl}_{3}\right) \delta 9.04(\mathrm{~s}, 1 \mathrm{H}), 8.33\left(\mathrm{~d},{ }^{3} \mathrm{~J}_{\mathrm{H}, \mathrm{H}}=8.5 \mathrm{~Hz}, 1 \mathrm{H}\right)$, $7.31\left(\mathrm{~d},{ }^{3} \mathrm{~J}_{\mathrm{H}, \mathrm{H}}=8.6 \mathrm{~Hz}, 1 \mathrm{H}\right), 3.84(\mathrm{~s}, 2 \mathrm{H}), 3.58(\mathrm{~s}, 3 \mathrm{H}), 2.36(\mathrm{~s}, 3 \mathrm{H}) ;{ }^{13} \mathrm{C} \mathrm{NMR}\left(101 \mathrm{MHz}, \mathrm{CDCl}_{3}\right)$ $\delta 164.8,135.2,134.7,132.2,130.1,123.4,122.6\left(\mathrm{q},{ }^{1} J_{\mathrm{C}, \mathrm{F}}=292 \mathrm{~Hz}\right), 114.6,85.1\left(\mathrm{p},{ }^{2} J_{\mathrm{C}, \mathrm{F}}=28.6\right.$ $\mathrm{Hz}), 54.9,21.2,0.1 ;{ }^{19} \mathrm{~F} \mathrm{NMR}\left(376 \mathrm{MHz}, \mathrm{CDCl}_{3}\right) \delta-69.40$ (s). Anal. Calcd for $\mathrm{C}_{13} \mathrm{H}_{12} \mathrm{~F}_{6} \mathrm{INO}_{2}(\%)$ C, 34.31; H, 2.66; N, 3.08. Found: C, 34.03; H, 2.69; N, 2.78.

4.7.2. $\quad \mathrm{N}$-[2-(1,1,1,3,3,3-Hexafluoro-2-methoxypropan-2-yl)-4-methoxyphenyl]-2-iodoacetamide (12b).

Yield: $91 \%$; mp $87-88^{\circ} \mathrm{C} ;{ }^{1} \mathrm{H}$ NMR $\left(400 \mathrm{MHz}, \mathrm{CDCl}_{3}\right) \delta 8.87(\mathrm{~s}, 1 \mathrm{H}), 8.31\left(\mathrm{~d},{ }^{3} \mathrm{~J}_{\mathrm{H}, \mathrm{H}}=8.9 \mathrm{~Hz}, 1 \mathrm{H}\right)$, $7.04(\mathrm{~d}, J=9.1 \mathrm{~Hz}, 1 \mathrm{H}), 7.03(\mathrm{~s}, 1 \mathrm{H}), 3.84(\mathrm{~s}, 2 \mathrm{H}), 3.81(\mathrm{~s}, 3 \mathrm{H}), 3.57(\mathrm{~s}, 3 \mathrm{H}) ;{ }^{13} \mathrm{C} \mathrm{NMR}(101 \mathrm{MHz}$, $\left.\mathrm{CDCl}_{3}\right) \delta 164.7,156.3,130.5,125.3,122.5\left(\mathrm{q},{ }^{1} J_{\mathrm{C}, \mathrm{F}}=290 . \mathrm{Hz}\right), 116.6,116.2,115.9,84.9\left(\mathrm{p},{ }^{2} J_{\mathrm{C}, \mathrm{F}}\right.$ $=29 \mathrm{~Hz}), 55.7,54.9,0.1 ;{ }^{19} \mathrm{~F} \mathrm{NMR}\left(376 \mathrm{MHz}, \mathrm{CDCl}_{3}\right) \delta-69.46$ (s). Anal. Calcd for $\mathrm{C}_{13} \mathrm{H}_{12} \mathrm{~F}_{6} \mathrm{INO}_{3}$

(\%) C, 33.14; H, 2.57; N, 2.97. Found: C, 33.10; H, 2.72; N, 3.18.

4.8. General procedure for synthesis of $\mathbf{1 3 .}$

A mixture of $12(4.4 \mathrm{mmol})$ and mesitylamine $(9.3 \mathrm{~mL}, 65.9 \mathrm{mmol})$ was stirred at r.t. for 4 days. After reaction completion, the mixture was treated with a $10 \%$ solution of $\mathrm{NaHCO}_{3}(60 \mathrm{~mL})$ and extracted with EtOAc $(3 \times 30 \mathrm{~mL})$. Combined organic layers were washed with brine and water and then dried over $\mathrm{MgSO}_{4}$ and concentrated under reduced pressure. The excess of $\mathrm{MesNH}_{2}$ was removed at $70^{\circ} \mathrm{C} / 0.05 \mathrm{mmHg}$. The residual solid was recrystallized from petroleum ether to yield the corresponding aminoacetamide $\mathbf{7}$ as a white solid. 
4.8.1. N-[2-(1,1,1,3,3,3-Hexafluoro-2-methoxypropan-2-yl)-4-methylphenyl]-2-(mesitylamino)acetamide (13a).

Yield: 85\%; mp 160-161 ${ }^{\circ} \mathrm{C} ;{ }^{1} \mathrm{H}$ NMR (400 MHz, acetone- $\left.d_{6}\right) \delta 10.12(\mathrm{~s}, 1 \mathrm{H}), 8.58\left(\mathrm{~d},{ }^{3} \mathrm{~J}_{\mathrm{H}, \mathrm{H}}=8.6\right.$ $\mathrm{Hz}, 1 \mathrm{H}), 7.40\left(\mathrm{~d},{ }^{3} J_{\mathrm{H}, \mathrm{H}}=8.6 \mathrm{~Hz}, 1 \mathrm{H}\right), 7.33(\mathrm{~s}, 1 \mathrm{H}), 6.82(\mathrm{~s}, 2 \mathrm{H}), 4.25\left(\mathrm{t},{ }^{3} J_{\mathrm{H}, \mathrm{H}}=8.0 \mathrm{~Hz}, 1 \mathrm{H}\right), 3.74$ $\left(\mathrm{d},{ }^{3} J_{\mathrm{H}, \mathrm{H}}=8.1 \mathrm{~Hz}, 2 \mathrm{H}\right), 3.59(\mathrm{~s}, 3 \mathrm{H}), 2.38(\mathrm{~s}, 3 \mathrm{H}), 2.30(\mathrm{~s}, 6 \mathrm{H}), 2.19(\mathrm{~s}, 3 \mathrm{H}) ;{ }^{13} \mathrm{C} \mathrm{NMR}(101 \mathrm{MHz}$, acetone- $\left.d_{6}\right) \delta 170.3,143.6,137.0,134.5,132.8,132.5,130.5,130.4,124.3,123.6\left(\mathrm{~d},{ }^{1} J_{\mathrm{C}, \mathrm{F}}=291\right.$ $\mathrm{Hz}), 114.9,85.9\left(\mathrm{p},{ }^{2} J_{\mathrm{C}, \mathrm{F}}=28 \mathrm{~Hz}\right), 55.4,53.8,20.9,20.6,18.4 ;{ }^{19} \mathrm{~F}$ NMR (376 MHz, acetone- $\left.d_{6}\right)$ $\delta$-69.99 (s). Anal. Calcd for $\mathrm{C}_{22} \mathrm{H}_{24} \mathrm{~F}_{6} \mathrm{~N}_{2} \mathrm{O}_{2}(\%) \mathrm{C}, 57.14 ; \mathrm{H}, 5.23 ; \mathrm{N}, 6.06$. Found: $\mathrm{C}, 57.17 ; \mathrm{H}$, $5.23 ; \mathrm{N}, 5.71$.

4.8.2. $\mathrm{N}$-[2-(1,1,1,3,3,3-Hexafluoro-2-methoxypropan-2-yl)-4-methoxyphenyl]-2-(mesitylamino)acetamide $(\mathbf{1 3 b})$.

Yield: 91\%; mp 137-138 ${ }^{\circ} \mathrm{C} ;{ }^{1} \mathrm{H}$ NMR (400 MHz, acetone- $\left.d_{6}\right) \delta 9.93(\mathrm{~s}, 1 \mathrm{H}), 8.53\left(\mathrm{~d},{ }^{3} J_{\mathrm{H}, \mathrm{H}}=9.2\right.$ $\mathrm{Hz}, 1 \mathrm{H}), 7.21(\mathrm{dd}, J=9.2,2.8 \mathrm{~Hz}, 1 \mathrm{H}), 7.02(\mathrm{~s}, 1 \mathrm{H}), 6.82(\mathrm{~s}, 2 \mathrm{H}), 4.24\left(\mathrm{t},{ }^{3} J_{\mathrm{H}, \mathrm{H}}=7.8 \mathrm{~Hz}, 1 \mathrm{H}\right), 3.85$ $(\mathrm{s}, 3 \mathrm{H}), 3.73\left(\mathrm{~d},{ }^{3} \mathrm{~J}_{\mathrm{H}, \mathrm{H}}=7.9 \mathrm{~Hz}, 2 \mathrm{H}\right), 3.59(\mathrm{~s}, 3 \mathrm{H}), 2.30(\mathrm{~s}, 6 \mathrm{H}), 2.19$ (s, 3H); ${ }^{13} \mathrm{C}$ NMR $(101 \mathrm{MHz}$, acetone- $\left.d_{6}\right) \delta 170.1,156.7,143.7,132.5,132.2,130.5,130.4,126.5,123.5\left(\mathrm{q},{ }^{1} J_{\mathrm{C}, \mathrm{F}}=292 \mathrm{~Hz}\right)$, 116.8, 116.7, 116.3, 86.5-85.0 (m), 56.0, 55.5, 53.7, 20.6, 18.4; ${ }^{19}$ F NMR (376 MHz, acetone-d6) $\delta$-70.06 (s). Anal. Calcd for $\mathrm{C}_{22} \mathrm{H}_{24} \mathrm{~F}_{6} \mathrm{~N}_{2} \mathrm{O}_{3}(\%) \mathrm{C}, 55.23 ; \mathrm{H}, 5.06 ; \mathrm{N}, 5.86$. Found: C, 55.42; H, $5.03 ; \mathrm{N}, 5.96$.

4.9. General procedure for synthesis of $\mathbf{1 4}$.

Aminoacetamide 13 (3.46 mmol) was dissolved in anhydrous toluene $(30 \mathrm{~mL})$, and $\mathrm{BH}_{3} \cdot \mathrm{SMe}_{2}$ (15.6 $\mathrm{mL}$ of $1 \mathrm{M}$ solution in THF, $15.6 \mathrm{mmol}$ ) was added dropwise under an argon atmosphere at r.t. The resulting mixture was stirred at $90^{\circ} \mathrm{C}$ for $3 \mathrm{~h}$. After cooling to r.t., $\mathrm{MeOH}$ was slowly added until ceasing of gas evolution. Then, a $10 \%$ solution $(60 \mathrm{~mL})$ of $\mathrm{HCl}$ was added and resulting mixture was extracted with EtOAc $(2 \times 20 \mathrm{~mL})$. The aqueous layer was separated, treated with $\mathrm{NaHCO}_{3}$, and extracted with EtOAc $(2 \times 20 \mathrm{~mL})$. Combined organic layers were washed with a saturated solution of $\mathrm{NaHCO}_{3}$, dried over $\mathrm{MgSO}_{4}$, and concentrated under reduced pressure. The crude product was recrystallized from petroleum ether to yield a beige solid.

4.9.1. $N^{1}$-[2-(1,1,1,3,3,3-Hexafluoro-2-methoxypropan-2-yl)-4-methylphenyl]-N²-mesitylethane1,2-diamine (14a).

Yield: 90\%; mp 53-54 ${ }^{\circ} \mathrm{C} ;{ }^{1} \mathrm{H}$ NMR $\left(400 \mathrm{MHz}, \mathrm{CDCl}_{3}\right) \delta 7.13\left(\mathrm{~d},{ }^{3} J_{\mathrm{H}, \mathrm{H}}=8.9 \mathrm{~Hz}, 1 \mathrm{H}\right), 7.11(\mathrm{~s}, 1 \mathrm{H})$, $6.86(\mathrm{~s}, 2 \mathrm{H}), 6.72\left(\mathrm{~d},{ }^{3} J_{\mathrm{H}, \mathrm{H}}=8.4 \mathrm{~Hz}, 1 \mathrm{H}\right), 5.70$ (br.s, $\left.1 \mathrm{H}\right), 3.54(\mathrm{~s}, 3 \mathrm{H}), 3.46-3.39(\mathrm{~m}, 2 \mathrm{H}), 3.20(\mathrm{t}$, $\left.{ }^{3} J_{\mathrm{H}, \mathrm{H}}=5.9 \mathrm{~Hz}, 2 \mathrm{H}\right), 2.30(\mathrm{~s}, 6 \mathrm{H}), 2.27(\mathrm{~s}, 3 \mathrm{H}), 2.25(\mathrm{~s}, 3 \mathrm{H}) ;{ }^{13} \mathrm{C} \mathrm{NMR}\left(101 \mathrm{MHz}, \mathrm{CDCl}_{3}\right) \delta 146.2$, 132.2, 130.8, 130.7, 129.7, 125.6, $123.0\left(\mathrm{q},{ }^{1} J_{\mathrm{C}, \mathrm{F}}=293 \mathrm{~Hz}\right), 112.8,108.7,85.7\left(\mathrm{p},{ }^{1} J_{\mathrm{C}, \mathrm{F}}=28.3 \mathrm{~Hz}\right)$, 54.6, 48.1, 44.7, 20.7, 20.6, 18.2; ${ }^{19} \mathrm{~F}$ NMR (376 MHz, $\left.\mathrm{CDCl}_{3}\right) \delta-69.46$ (s). Anal. Calcd for $\mathrm{C}_{22} \mathrm{H}_{26} \mathrm{~F}_{6} \mathrm{~N}_{2} \mathrm{O}(\%) \mathrm{C}, 58.92 ; \mathrm{H}, 5.84 ; \mathrm{N}, 6.25$. Found: C, 58.81; H, 5.87; N, 5.96. 
mesitylethane-1,2-diamine $(\mathbf{1 4 b})$.

Yield: $72 \%$; mp 56-57 ${ }^{\circ}$; ${ }^{1} \mathrm{H}$ NMR $\left(400 \mathrm{MHz}, \mathrm{CDCl}_{3}\right) \delta 6.97\left(\mathrm{~d},{ }^{3} \mathrm{~J}_{\mathrm{H}, \mathrm{H}}=8.9 \mathrm{~Hz}, 1 \mathrm{H}\right), 6.95(\mathrm{~s}, 1 \mathrm{H})$, $6.86(\mathrm{~s}, 2 \mathrm{H}), 6.76\left(\mathrm{~d},{ }^{3} J_{\mathrm{H}, \mathrm{H}}=8.8 \mathrm{~Hz}, 1 \mathrm{H}\right), 5.54(\mathrm{~s}, 1 \mathrm{H}), 3.77(\mathrm{~s}, 3 \mathrm{H}), 3.56(\mathrm{~s}, 3 \mathrm{H}), 3.37\left(\mathrm{dd},{ }^{3} J_{\mathrm{H}, \mathrm{H}}=\right.$ 10.9, $5.3 \mathrm{~Hz}, 2 \mathrm{H}), 3.19\left(\mathrm{t},{ }^{3} \mathrm{~J}_{\mathrm{H}, \mathrm{H}}=5.8 \mathrm{~Hz}, 2 \mathrm{H}\right), 2.93$ (br.s, $\left.1 \mathrm{H}\right), 2.29$ (s, 6H), $2.26(\mathrm{~s}, 3 \mathrm{H}) ;{ }^{13} \mathrm{C} \mathrm{NMR}$ $\left(101 \mathrm{MHz}, \mathrm{CDCl}_{3}\right) \delta 150.8,143.1,142.9,132.3,130.7,129.7,122.9\left(\mathrm{q},{ }^{1} J_{\mathrm{C}, \mathrm{F}}=292 \mathrm{~Hz}\right), 117.5$, $116.5,113.9,109.6,85.6\left(\mathrm{p},{ }^{2} J_{\mathrm{C}, \mathrm{F}}=28 \mathrm{~Hz}\right), 56.0,54.8,48.1,45.5,20.7,18.2 ;{ }^{19} \mathrm{~F} \mathrm{NMR}(376 \mathrm{MHz}$, $\left.\mathrm{CDCl}_{3}\right) \delta-69.50$ (s). Anal. Calcd for $\mathrm{C}_{22} \mathrm{H}_{26} \mathrm{~F}_{6} \mathrm{~N}_{2} \mathrm{O}_{2}(\%) \mathrm{C}, 56.89 ; \mathrm{H}, 5.64 ; \mathrm{N}, 6.03$. Found: C, $57.08 ; \mathrm{H}, 5.65 ; \mathrm{N}, 6.15$.

\subsection{General procedure for synthesis of 15.}

Acetic formic anhydride $(360 \mu \mathrm{L}, 4.13 \mathrm{mmol})$ was added dropwise to the solution of 14 (2.06 mmol) in $\mathrm{CH}_{2} \mathrm{Cl}_{2}(13 \mathrm{~mL})$. After homogenization, the reaction mixture was allowed to stir for 30 min at r.t. Then, $40 \mathrm{~mL}$ of water was added and the mixture was extracted with $\mathrm{CH}_{2} \mathrm{Cl}_{2}(2 \times 30 \mathrm{~mL})$. Combined organic layers were washed with a saturated solution of $\mathrm{NaHCO}_{3}$ and water, dried over $\mathrm{MgSO}_{4}$, filtered, and concentrated under reduced pressure. The crude product was purified by column chromatography using EtOAc/petroleum ether (1:4) as eluent to yield colorless oil.

4.10.1. $\quad N-(2-\{[2-(1,1,1,3,3,3-H e x a f l u o r o-2-m e t h o x y p r o p a n-2-y l)-4-m e t h y l p h e n y l] a m i n o\}$ ethyl)$N$-mesitylformamide (15a).

Yield: $98 \%$; $\mathrm{mp} 101-102^{\circ} \mathrm{C}$; mixture of two rotamers $85: 15$, signals of major product are given: ${ }^{1} \mathrm{H}$ NMR $\left(400 \mathrm{MHz}, \mathrm{CDCl}_{3}\right) \delta 8.06(\mathrm{~s}, 1 \mathrm{H}), 7.13\left(\mathrm{~d},{ }^{3} J_{\mathrm{H}, \mathrm{H}}=8.5 \mathrm{~Hz}, 1 \mathrm{H}\right), 7.10(\mathrm{~s}, 1 \mathrm{H}), 6.97(\mathrm{~s}, 2 \mathrm{H})$, $6.75\left(\mathrm{~d},{ }^{3} J_{\mathrm{H}, \mathrm{H}}=8.5 \mathrm{~Hz}, 1 \mathrm{H}\right), 5.80($ br.s, $1 \mathrm{H}), 3.81\left(\mathrm{t},{ }^{3} J_{\mathrm{H}, \mathrm{H}}=7.0 \mathrm{~Hz}, 2 \mathrm{H}\right), 3.52(\mathrm{~s}, 3 \mathrm{H}), 3.37\left(\mathrm{t},{ }^{3} J_{\mathrm{H}, \mathrm{H}}\right.$ $=7.0 \mathrm{~Hz}, 2 \mathrm{H}), 2.32(\mathrm{~s}, 3 \mathrm{H}), 2.25(\mathrm{~s}, 3 \mathrm{H}), 2.21(\mathrm{~s}, 6 \mathrm{H}) ;{ }^{13} \mathrm{C} \mathrm{NMR}\left(75 \mathrm{MHz}, \mathrm{CDCl}_{3}\right) \delta 164.2,145.3$, 138.7, 136.6, 136.0, 132.4, 130.6, 129.9, 126.2, $123.0\left(\mathrm{q},{ }^{1} J_{\mathrm{C}, \mathrm{F}}=292 \mathrm{~Hz}\right), 113.1,109.3,86.5-84.7$ (m), 54.7, 45.7, 42.6, 21.0, 20.6, 18.3; $\left.{ }^{19} \mathrm{~F} \mathrm{NMR} \mathrm{(376} \mathrm{MHz,} \mathrm{CDCl}_{3}\right) \delta-69.55$ (s). Anal. Calcd for $\mathrm{C}_{23} \mathrm{H}_{26} \mathrm{~F}_{6} \mathrm{~N}_{2} \mathrm{O}_{2}$ (\%) C, 57.98; H, 5.50; N, 5.88. Found: C, 57.69; H, 5.63; N, 6.04.

4.10.2. $N$-(2-\{[2-(1,1,1,3,3,3-Hexafluoro-2-methoxypropan-2-yl)-4-methoxyphenyl]amino\}ethyl)$N$-mesitylformamide (15b).

Yield: 99\%; mp $115-116^{\circ} \mathrm{C}$; mixture of two rotamers 90:10, signals of major product are given: ${ }^{1} \mathrm{H}$ $\operatorname{NMR}\left(400 \mathrm{MHz}, \mathrm{CDCl}_{3}\right) \delta 8.06(\mathrm{~s}, 1 \mathrm{H}), 6.97(\mathrm{~s}, 2 \mathrm{H}), 6.95\left(\mathrm{~d},{ }^{3} J_{\mathrm{H}, \mathrm{H}}=9.2 \mathrm{~Hz}, 1 \mathrm{H}\right), 6.91(\mathrm{~s}, 1 \mathrm{H})$, $6.81\left(\mathrm{~d},{ }^{3} J_{\mathrm{H}, \mathrm{H}}=9.0 \mathrm{~Hz}, 1 \mathrm{H}\right), 5.84(\mathrm{br} . \mathrm{s}, 1 \mathrm{H}), 3.80\left(\mathrm{t},{ }^{3} J_{\mathrm{H}, \mathrm{H}}=7.0 \mathrm{~Hz}, 2 \mathrm{H}\right), 3.74(\mathrm{~s}, 3 \mathrm{H}), 3.52(\mathrm{~s}, 3 \mathrm{H})$, $3.36\left(\mathrm{t},{ }^{3} J_{\mathrm{H}, \mathrm{H}}=7.0 \mathrm{~Hz}, 2 \mathrm{H}\right), 2.31(\mathrm{~s}, 3 \mathrm{H}), 2.21(\mathrm{~s}, 6 \mathrm{H}) ;{ }^{13} \mathrm{C} \mathrm{NMR}\left(75 \mathrm{MHz}, \mathrm{CDCl}_{3}\right) \delta 164.2,151.2$, 141.8, 138.7, 136.6, 136.0, 129.9, 122.9 (q, $\left.{ }^{1} J_{\mathrm{C}, \mathrm{F}}=293 \mathrm{~Hz}\right), 117.6,116.6,114.3,110.2,85.5$ (p, $\left.{ }^{2} J_{\mathrm{C}, \mathrm{F}}=28 \mathrm{~Hz}\right), 56.0,54.8,45.8,43.0,21.0,18.3 ;{ }^{19} \mathrm{~F} \mathrm{NMR}\left(376 \mathrm{MHz}, \mathrm{CDCl}_{3}\right) \delta-69.61$ (s). Anal. Calcd for $\mathrm{C}_{23} \mathrm{H}_{26} \mathrm{~F}_{6} \mathrm{~N}_{2} \mathrm{O}_{3}(\%)$ C, 56.10; H, 5.32; N, 5.69. Found: C, 56.11; H, 5.55; N, 5.91. 4.11. General procedure for synthesis of 16. 
Triflic acid $(180 \mu \mathrm{L}, 2.0 \mathrm{mmol})$ was added to the solution of $15(2.0 \mathrm{mmol})$ in toluene $(50 \mathrm{~mL})$, and the mixture was stirred at r.t. for $15 \mathrm{~min}$. Then, triflic anhydride $(340 \mu \mathrm{L}, 2.0 \mathrm{mmol})$ was added, and the reaction mixture was heated at $65^{\circ} \mathrm{C}$ for $1.5 \mathrm{~h}$. DIPEA $(1.04 \mathrm{~mL}, 6.0 \mathrm{mmol})$ was added, and the reaction mixture was heated at $80^{\circ} \mathrm{C}$ for another $1.5 \mathrm{~h}$. After cooling to r.t., $50 \mathrm{~mL}$ of water was added and the mixture was extracted with $\mathrm{CH}_{2} \mathrm{Cl}_{2}(3 \times 30 \mathrm{~mL})$. The combined organic layers were dried using $\mathrm{MgSO}_{4}$, filtered, and concentrated under reduced pressure. The crude product was recrystallized from petroleum ether to yield a beige solid.

4.11.1. 1-[2-(1,1,1,3,3,3-Hexafluoro-2-methoxypropan-2-yl)-4-methylphenyl]-3-mesityl-4,5dihydro-1H-imidazol-3-ium triflate (16a).

Yield: 85\%; mp 167-169 ${ }^{\circ} \mathrm{C} ;{ }^{1} \mathrm{H}$ NMR $\left(400 \mathrm{MHz}, \mathrm{CDCl}_{3}\right) \delta 8.08(\mathrm{~s}, 1 \mathrm{H}), 7.74\left(\mathrm{~d},{ }^{3} \mathrm{~J}_{\mathrm{H}, \mathrm{H}}=8.1 \mathrm{~Hz}\right.$, $1 \mathrm{H}), 7.53(\mathrm{~s}, 1 \mathrm{H}), 7.46\left(\mathrm{~d},{ }^{3} J_{\mathrm{H}, \mathrm{H}}=8.0 \mathrm{~Hz}, 1 \mathrm{H}\right), 6.95(\mathrm{~s}, 2 \mathrm{H}), 4.64\left(\mathrm{t},{ }^{3} J_{\mathrm{H}, \mathrm{H}}=10.6 \mathrm{~Hz}, 2 \mathrm{H}\right), 4.40(\mathrm{t}$, $\left.{ }^{3} J_{\mathrm{H}, \mathrm{H}}=10.6 \mathrm{~Hz}, 2 \mathrm{H}\right), 3.61(\mathrm{~s}, 3 \mathrm{H}), 2.44(\mathrm{~s}, 3 \mathrm{H}), 2.34(\mathrm{~s}, 6 \mathrm{H}), 2.29(\mathrm{~s}, 3 \mathrm{H}) ;{ }^{13} \mathrm{C} \mathrm{NMR}(101 \mathrm{MHz}$, $\left.\mathrm{CDCl}_{3}\right) \delta 159.7,141.9,141.0,135.1,133.9,132.3,132.0,131.6,130.2,130.1,125.0,122.3(\mathrm{q}$, $\left.{ }^{1} J_{\mathrm{C}, \mathrm{F}}=290 \mathrm{~Hz}\right), 120.7\left(\mathrm{q},{ }^{1} J_{\mathrm{C}, \mathrm{F}}=320 \mathrm{~Hz}\right), 84.2-82.8(\mathrm{~m}), 55.7,55.1,51.8,21.6,21.1,17.7 ;{ }^{19} \mathrm{~F}$ NMR (376 MHz, $\left.\mathrm{CDCl}_{3}\right) \delta-69.24(\mathrm{~s}, 6 \mathrm{~F}),-78.59(\mathrm{~s}, 3 \mathrm{~F})$. Anal. Calcd for $\mathrm{C}_{24} \mathrm{H}_{25} \mathrm{~F}_{9} \mathrm{~N}_{2} \mathrm{O}_{4} \mathrm{~S}(\%) \mathrm{C}$, 47.37; H, 4.14; N, 4.60. Found: C, 47.61; H, 4.14; N, 4.58.

4.11.2. 1-[2-(1,1,1,3,3,3-Hexafluoro-2-methoxypropan-2-yl)-4-methoxyphenyl]-3-mesityl-4,5dihydro-1H-imidazol-3-ium triflate (16b).

Yield: $83 \%$; mp $171-173{ }^{\circ} \mathrm{C}$; ${ }^{1} \mathrm{H}$ NMR $\left(400 \mathrm{MHz}, \mathrm{CDCl}_{3}\right) \delta 8.04(\mathrm{~s}, 1 \mathrm{H}), 7.85\left(\mathrm{~d},{ }^{3} \mathrm{~J}_{\mathrm{H}, \mathrm{H}}=8.7 \mathrm{~Hz}\right.$, $1 \mathrm{H}), 7.25(\mathrm{~s}, 1 \mathrm{H}), 7.15\left(\mathrm{dd}, J_{\mathrm{H}, \mathrm{H}}=8.7,2.6 \mathrm{~Hz}, 1 \mathrm{H}\right), 6.95(\mathrm{~s}, 2 \mathrm{H}), 4.61\left(\mathrm{t},{ }^{3} J_{\mathrm{H}, \mathrm{H}}=10.5 \mathrm{~Hz}, 2 \mathrm{H}\right), 4.39$ $\left(\mathrm{t},{ }^{3} J_{\mathrm{H}, \mathrm{H}}=10.4 \mathrm{~Hz}, 2 \mathrm{H}\right), 3.84(\mathrm{~s}, 3 \mathrm{H}), 3.61(\mathrm{~s}, 3 \mathrm{H}), 2.34(\mathrm{~s}, 6 \mathrm{H}), 2.29(\mathrm{~s}, 3 \mathrm{H}) ;{ }^{13} \mathrm{C} \mathrm{NMR}(101 \mathrm{MHz}$, $\left.\mathrm{CDCl}_{3}\right) \delta 161.0,159.9,141.0,135.1,133.9,130.2,130.1,126.9,126.7,122.3\left(\mathrm{q},{ }^{1} J_{\mathrm{C}, \mathrm{F}}=291 \mathrm{~Hz}\right)$, $120.7\left(\mathrm{q},{ }^{1} J_{\mathrm{C}, \mathrm{F}}=320 \mathrm{~Hz}\right), 118.3,116.8,84.2-82.6(\mathrm{~m}), 56.0,55.8,55.1,51.8,21.1,17.7 ;{ }^{19} \mathrm{~F} \mathrm{NMR}$ $\left(376 \mathrm{MHz}, \mathrm{CDCl}_{3}\right) \delta-69.27(\mathrm{~s}, 6 \mathrm{~F}),-78.55$ (s, 3F). Anal. Calcd for $\mathrm{C}_{24} \mathrm{H}_{25} \mathrm{~F}_{9} \mathrm{~N}_{2} \mathrm{O} 5 \mathrm{~S}(\%) \mathrm{C}, 46.16$; H, 4.03; N, 4.49. Found: C, 46.21; H, 4.06; N, 4.51.

\subsection{General procedure for synthesis of Grubbs-type catalysts 3 and 4.}

In a flame-dried Schlenk flask, imidazolinium salt $16(0.49 \mathrm{mmol})$ was mixed with $20 \mathrm{~mL}$ of anhydrous toluene. The resulting mixture was cooled to $0^{\circ} \mathrm{C}$ and degassed three times, and then KHMDS ( $1 \mathrm{~mL}$ of $0.5 \mathrm{M}$ solution in toluene, $0.50 \mathrm{mmol}$ ) was added to the mixture under an argon atmosphere. The reaction mixture was stirred for $30 \mathrm{~min}$ at r.t.; then Grubbs' catalyst $\mathbf{G}-\mathbf{I}(0.33 \mathrm{~g}$, $0.40 \mathrm{mmol}$ ) was added and mixture was stirred for $2 \mathrm{~h}$. During this time, the reaction mixture changed color from violet to red-brown. Once complete (TLC-control), solvents were removed from the reaction mixture under reduced pressure, and the resulting substance was purified by column chromatography in a gradient manner using EtOAc/petroleum ether (1:8-1:3) as eluent 
under an argon atmosphere. The resulting solid was recrystallized from $\mathrm{MeOH}$ to yield $\mathbf{1 1}$ as a brown solid.

4.12.1.

Benzylidene(dichloro)\{1-[2-(1,1,1,3,3,3-hexafluoro-2-methoxypropan-2-yl)-4methylphenyl]-3-mesityl-4,5-dihydroimidazol-2-ylidene)(triphenylphosphine)ruthenium(II) (3).

Yield: $46 \%$; mixture of two rotamers $4: 1$ at $60^{\circ} \mathrm{C}$, signals of major product are given: ${ }^{1} \mathrm{H}$ NMR $\left(400 \mathrm{MHz}\right.$, toluene- $\left.d_{8}, 60^{\circ} \mathrm{C}\right) \delta 19.62(\mathrm{~s}, 1 \mathrm{H}, \mathrm{CHAr}), 9.47\left(\mathrm{~d}, J_{\mathrm{H}, \mathrm{H}}=7.9 \mathrm{~Hz}, 1 \mathrm{H}, \mathrm{ArH}\right), 8.25(\mathrm{~s}, 1 \mathrm{H}$, $\operatorname{ArH}), 7.74(\mathrm{~s}, 1 \mathrm{H}, \operatorname{Ar} H), 7.35\left(\mathrm{~d}, J_{\mathrm{H}, \mathrm{H}}=7.5 \mathrm{~Hz}, 1 \mathrm{H}, \operatorname{ArH}\right), 7.20-7.10(\mathrm{~m}, 2 \mathrm{H}, \operatorname{ArH}), 6.94-6.72(\mathrm{~m}$, 2H, $\mathrm{ArH}$ ), 6.34 (br.s, 1H, $\mathrm{ArH}$ ), 6.01 (m, 1H, $\mathrm{ArH}), 4.43$ (s, 1H, $\left.\mathrm{CH}_{2} \mathrm{CH}_{2}\right), 4.12$ (s, 3H, $\left.\mathrm{C}\left(\mathrm{CF}_{3}\right)_{2} \mathrm{OCH}_{3}\right), 3.78\left(\mathrm{~s}, 1 \mathrm{H}, \mathrm{CH}_{2} \mathrm{CH}_{2}\right), 3.39$ (s, $\left.1 \mathrm{H}, \mathrm{CH}_{2} \mathrm{CH}_{2}\right), 3.24$ (s, 1H, $\left.\mathrm{CH}_{2} \mathrm{CH}_{2}\right), 2.48$ (s, , $\left.\mathrm{CH}_{3}\right), 2.74-0.92\left(\mathrm{~m}, 42 \mathrm{H}, \mathrm{PCy}, \mathrm{CH}_{3}\right) ;{ }^{13} \mathrm{C}$ NMR $\left(126 \mathrm{MHz}\right.$, toluene- $\left.d_{8}, 60^{\circ} \mathrm{C}\right) \delta 298.2,223.4$, 152.4, 139.6, 142.2, 138.9, 138.7, 137.0, 136.7, 135.4, 131.9 (br.s), 130.6, 130.4, 129.7, 129.5, $128.3,123.6\left(\mathrm{q},{ }^{1} J_{\mathrm{C}, \mathrm{F}}=289 \mathrm{~Hz}\right), 123.4\left(\mathrm{q},{ }^{1} J_{\mathrm{C}, \mathrm{F}}=290 \mathrm{~Hz}\right), 117.6,117.3,85.6-84.1(\mathrm{~m}), 58.5,54.9$, 52.2, 29.8, 29.5, 29.4, 28.1-28.0 (m), 26.8, 21.0, 19.3, 19.0; ${ }^{19} \mathrm{~F}$ NMR (376 MHz, toluene- $\left.d 8,60^{\circ} \mathrm{C}\right)$ $\delta$-65.29 (br.s, 3F), -71.15 (br.s, 3F); ${ }^{31}$ P NMR (202 MHz, toluene- $\left.d 8,60^{\circ} \mathrm{C}\right) \delta 24.2$ (s). Anal. Calcd for $\mathrm{C}_{48} \mathrm{H}_{63} \mathrm{Cl}_{2} \mathrm{~F}_{6} \mathrm{~N}_{2} \mathrm{OPRu}(\%) \mathrm{C}, 57.60 ; \mathrm{H}, 6.34 ; \mathrm{N}, 2.80$. Found: C, 57.39; H, 6.47; N, 2.84. 4.12.2. Benzylidene(dichloro) $\{1-[2-(1,1,1,3,3,3$-hexafluoro-2-methoxypropan-2-yl)-4methoxyphenyl]-3-mesityl-4,5-dihydroimidazol-2-ylidene\}(triphenylphosphine)ruthenium(II) (4). Yield: $48 \%$; mixture of two rotamers $4: 1$ at $50^{\circ} \mathrm{C}$, signals of major product are given: ${ }^{1} \mathrm{H}$ NMR (400 MHz, toluene- $d 8,50^{\circ} \mathrm{C}$ ) $\delta 19.59$ (s, $1 \mathrm{H}, \mathrm{CHAr}$ ), 9.62 (br.s, $1 \mathrm{H}, \operatorname{Ar} H$ ), 9.47 (br.s, $1 \mathrm{H}, \operatorname{Ar} H$ ), $7.61(\mathrm{~s}, 1 \mathrm{H}, \operatorname{Ar} H), 7.21\left(\mathrm{t}, J_{\mathrm{H}, \mathrm{H}}=7.3 \mathrm{~Hz}, 1 \mathrm{H}, \operatorname{Ar} H\right), 7.12\left(\mathrm{dd}, J_{\mathrm{H}, \mathrm{H}}=8.8,2.6 \mathrm{~Hz}, 1 \mathrm{H}, \operatorname{Ar} H\right), 7.08$ -

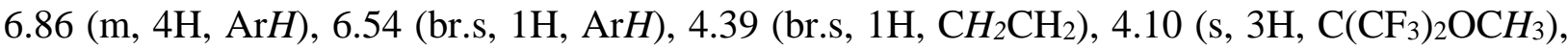
3.76 (br.s, $1 \mathrm{H}, \mathrm{CH}_{2} \mathrm{CH}_{2}$ ), 3.43 (s, $3 \mathrm{H}, \mathrm{ArOCH}_{3}$ ), 3.38 (br.s, $1 \mathrm{H}, \mathrm{CH}_{2} \mathrm{CH}_{2}$ ), 3.25 (s, $1 \mathrm{H}, \mathrm{CH}_{2} \mathrm{CH}_{2}$ ), 2.77-0.81 (m, 42H, $\left.\mathrm{PCy}_{3}, \mathrm{CH}_{3}\right) ;{ }^{13} \mathrm{C}$ NMR $\left(126 \mathrm{MHz}\right.$, toluene- $\left.d_{8}, 50^{\circ} \mathrm{C}\right) \delta 298.2,223.3,159.3$, 152.4, 139.8, 138.9, 138.7, 137.0, 136.7, 135.4, 132.0 (br.s), 130.6, 130.4, 129.7, 129.5, 128.3, $123.63\left(\mathrm{q},{ }^{1} J_{\mathrm{C}, \mathrm{F}}=284 \mathrm{~Hz}\right), 123.58\left(\mathrm{q},{ }^{1} J_{\mathrm{C}, \mathrm{F}}=288 \mathrm{~Hz}\right), 117.6,117.3$, 85.4-84.1 (m), 58.5, 56.3, 54.9, 52.2, 29.8, 29.4, 28.2-28.1 (m), 26.8, 21.0, 19.3, 18.9; ${ }^{19} \mathrm{~F}$ NMR (471 MHz, toluene- $\left.d 8,50{ }^{\circ} \mathrm{C}\right)$ $\delta$-65.50 (br.s, 3F), -71.13 (br.s,, 3F); ${ }^{31} \mathrm{P}$ NMR (202 MHz, toluene- $\left.d 8,50{ }^{\circ} \mathrm{C}\right) \delta 24.6$ (s). Anal. Calcd for $\mathrm{C}_{48} \mathrm{H}_{63} \mathrm{Cl}_{2} \mathrm{~F}_{6} \mathrm{~N}_{2} \mathrm{O}_{2} \mathrm{PRu}(\%)$ C, 56.69; H, 6.24; N, 2.75. Found: C, 56.68; H, 6.42; N, 2.77. 4.13. General procedure for synthesis of Hoveyda-Grubbs-type catalysts 5 and $\mathbf{6}$.

In a flame-dried Schlenk flask, imidazolinium salt $16(0.40 \mathrm{mmol})$ was mixed with $9 \mathrm{~mL}$ of anhydrous toluene. The resulting mixture was cooled to $0^{\circ} \mathrm{C}$ and degassed three times; then KHMDS ( $840 \mu \mathrm{L}$ of $0.5 \mathrm{M}$ solution in toluene, $0.42 \mathrm{mmol}$ ) was added to the mixture under an argon atmosphere. The reaction mixture was stirred for $30 \mathrm{~min}$ at r.t.; then Hoveyda-Grubbs catalyst first generation $\mathbf{H}-\mathbf{I}(0.20 \mathrm{~g}, 0.30 \mathrm{mmol})$ was added and mixture was stirred for $40 \mathrm{~min}$ at $80^{\circ} \mathrm{C}$. During this time, the reaction mixture changed color from brown to dark green. Once 
complete, solvents were removed from the reaction mixture under reduced pressure, and the resulting substance was purified by column chromatography using EtOAc/petroleum ether (1:3) as eluent to yield. Hoveyda-type catalyst as a green solid. Suitable for X-ray crystals of $\mathbf{5}$ and $\mathbf{6}$ were grown by slow diffusion of hexane vapors in $\mathrm{CH}_{2} \mathrm{Cl}_{2}$ solution.

4.13.1. Dichloro\{1-[2-(1,1,1,3,3,3-hexafluoro-2-methoxypropan-2-yl)-4-methylphenyl]-3mesityl-4,5-dihydroimidazol-2-ylidene)(2-isopropoxybenzylidene)ruthenium(II) (5).

Yield: 74\%; ${ }^{1} \mathrm{H}$ NMR $\left(500 \mathrm{MHz}\right.$, toluene- $\left.d 8,80^{\circ} \mathrm{C}\right) \delta 16.91$ (s, $\left.1 \mathrm{H}, \mathrm{CHAr}\right), 9.14$ (br.s, $\left.1 \mathrm{H}, \mathrm{ArH}\right)$, 7.78 (s, 1H, ArH), 7.21 (br.s, 1H, ArH), 7.10 (m, 1H, ArH), 6.94 (dd, $J_{\mathrm{H}, \mathrm{H}}=7.5,1.1 \mathrm{~Hz}, 1 \mathrm{H}, \mathrm{Ar} H$ ), $6.89(\mathrm{~s}, 1 \mathrm{H}, \operatorname{Ar} H), 6.82(\mathrm{~s}, 1 \mathrm{H}, \operatorname{Ar} H), 6.59\left(\mathrm{t},{ }^{3} J_{H, H}=7.4 \mathrm{~Hz}, 1 \mathrm{H}, \operatorname{Ar} H\right), 6.43\left(\mathrm{~d},{ }^{3} J_{\mathrm{H}, \mathrm{H}}=8.3 \mathrm{~Hz}\right.$, $1 \mathrm{H}, \mathrm{ArH}), 4.56\left(\right.$ hept, $\left.{ }^{3} J_{\mathrm{H}, \mathrm{H}}=6.3 \mathrm{~Hz}, 1 \mathrm{H}, \mathrm{O}^{i} \mathrm{Pr}, \mathrm{CH}\right), 4.51\left(\mathrm{~m}, 1 \mathrm{H}, \mathrm{H}_{2} \mathrm{I} \mathrm{CH} \mathrm{CH}_{2}\right), 3.86(\mathrm{~s}, 3 \mathrm{H}$, $\left.\mathrm{C}\left(\mathrm{CF}_{3}\right)_{2} \mathrm{OCH}_{3}\right), 3.69\left(\mathrm{q}, J_{\mathrm{H}, \mathrm{H}}=9.9 \mathrm{~Hz}, 1 \mathrm{H}, \mathrm{H}_{2} \mathrm{I} \mathrm{CH} \mathrm{CH}_{2}\right), 3.48\left(\mathrm{q}, J_{\mathrm{H}, \mathrm{H}}=9.9 \mathrm{~Hz}, 1 \mathrm{H}_{2} \mathrm{H}_{2} \mathrm{I} \mathrm{CH}_{2} \mathrm{CH}_{2}\right.$ ), $3.42\left(\mathrm{q}, J_{\mathrm{H}, \mathrm{H}}=9.3 \mathrm{~Hz}, 1 \mathrm{H}, \mathrm{H}_{2} \mathrm{I} \mathrm{CH} \mathrm{CH}_{2}\right.$ ), 2.51 (s, 3H, $\left.\mathrm{CH}_{3}\right), 2.37$ (s, 3H, CH3), 2.21 (s, 3H, $\mathrm{CH}_{3}$ ), 2.16 (s, 3H, CH3), 1.39 (s, 3H, O $\left.{ }^{i} \mathrm{Pr} \mathrm{CH}_{3}\right), 1.31\left(\mathrm{~d},{ }^{3} \mathrm{~J}_{\mathrm{H}, \mathrm{H}}=6.1 \mathrm{~Hz}, 3 \mathrm{H}, \mathrm{O}^{i} \mathrm{Pr} \mathrm{CH}_{3}\right) ;{ }^{13} \mathrm{C} \mathrm{NMR}(126$ $\mathrm{MHz}$, toluene- $\left.d 8,80^{\circ} \mathrm{C}\right) \delta 294.0,216.8,153.3,145.6,142.4,138.8,138.7,137.8,134.1$ (br.s), 130.4, 130.1, 130.0, 129.5, $123.8\left(\mathrm{q},{ }^{2} J_{\mathrm{C}, \mathrm{F}}=289 \mathrm{~Hz}\right), 123.4\left(\mathrm{q},{ }^{2} J_{\mathrm{C}, \mathrm{F}}=289 \mathrm{~Hz}\right), 122.4,122.3,113.4$, 85.4 (hept, ${ }^{1} J_{\mathrm{C}, \mathrm{F}}=27 \mathrm{~Hz}$ ), 75.1, 58.0, 54.6 (br.s), 53.1 (br.s), 22.4, 22.2, 22.1, 21.9; ${ }^{19} \mathrm{~F}$ NMR (471 $\mathrm{MHz}$, toluene- $d 8,60^{\circ} \mathrm{C}$ ) $\delta-65.6$ (br.s, 3F), -71.7 (br.s, 3F). Anal. Calcd for $\mathrm{C}_{33} \mathrm{H}_{36} \mathrm{Cl}_{2} \mathrm{~F}_{6} \mathrm{~N}_{2} \mathrm{O}_{2} \mathrm{Ru}$ (\%) C, 50.90; H, 4.66; N, 3.60. Found: C, 50.78; H, 4.89; N, 3.42. CCDC 1543696 contains the supplementary crystallographic data for this paper. These data can be obtained free of charge from The Cambridge Crystallographic Data Centre via http://www.ccdc.cam.ac.uk.

4.13.2. Dichloro\{1-[2-(1,1,1,3,3,3-hexafluoro-2-methoxypropan-2-yl)-4-methoxyphenyl]-3-

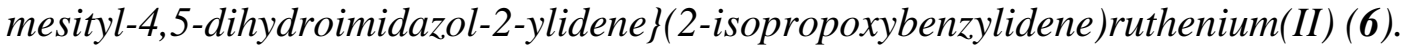

Yield: 71\%; ${ }^{1} \mathrm{H}$ NMR (500 MHz, toluene- $\left.d 8,80^{\circ} \mathrm{C}\right) \delta 16.92$ (br.s, $1 \mathrm{H}, \mathrm{CHAr}$ ), 9.13 (br.s, $1 \mathrm{H}, \mathrm{ArH}$ ), $7.61(\mathrm{~s}, 1 \mathrm{H}, \operatorname{Ar} H), 7.10(\mathrm{~m}, 1 \mathrm{H}, \operatorname{Ar} H), 6.98\left(\mathrm{dd}, J_{\mathrm{H}, \mathrm{H}}=1.5,7.4 \mathrm{~Hz}, 1 \mathrm{H}, \operatorname{Ar} H\right), 6.92$ (br.s, $1 \mathrm{H}, \operatorname{Ar} H$ ), $6.89(\mathrm{~s}, 1 \mathrm{H}, \operatorname{ArH}), 6.83(\mathrm{~s}, 1 \mathrm{H}, \operatorname{Ar} H), 6.58\left(\mathrm{t},{ }^{3} J_{\mathrm{H}, \mathrm{H}}=7.4 \mathrm{~Hz}, 1 \mathrm{H}\right), 6.42\left(\mathrm{~d},{ }^{3} J_{\mathrm{H}, \mathrm{H}}=8.3 \mathrm{~Hz}, 1 \mathrm{H}\right.$, $\mathrm{ArH}), 4.56\left(\right.$ hept $\left.,{ }^{3} J_{\mathrm{H}, \mathrm{H}}=6.1 \mathrm{~Hz}, 1 \mathrm{H}, \mathrm{O}^{i} \mathrm{Pr} \mathrm{CH}\right), 4.48\left(\mathrm{td}, J_{\mathrm{H}, \mathrm{H}}=9.6,3.2 \mathrm{~Hz}, 1 \mathrm{H}, \mathrm{H}_{2} \mathrm{I} \mathrm{CH} \mathrm{CH}_{2}\right.$ ), $3.85\left(\mathrm{~s}, 3 \mathrm{H}, \mathrm{C}\left(\mathrm{CF}_{3}\right)_{2} \mathrm{OCH}_{3}\right), 3.70\left(\mathrm{~m}, 1 \mathrm{H}, \mathrm{H}_{2} \mathrm{I} \mathrm{CH} \mathrm{CH}_{2}\right), 3.48\left(\mathrm{~m}, 1 \mathrm{H}, \mathrm{H}_{2} \mathrm{I} \mathrm{CH} \mathrm{CH}_{2}\right), 3.40(\mathrm{~m}, 1 \mathrm{H}$, $\mathrm{H}_{2} \mathrm{I} \mathrm{CH}_{2} \mathrm{CH}_{2}$ ), 3.38 (s, 3H, $\left.\mathrm{ArOCH}_{3}\right), 2.52$ (s, 3H, CH $), 2.39$ (s, 3H, CH $), 2.22\left(\mathrm{~s}, 3 \mathrm{H}, \mathrm{CH}_{3}\right), 1.32$ $\left(\mathrm{d},{ }^{3} J_{\mathrm{H}, \mathrm{H}}=6.1 \mathrm{~Hz}, 6 \mathrm{H}, \mathrm{O}^{i} \mathrm{Pr} \mathrm{CH}_{3}\right) ;{ }^{13} \mathrm{C} \mathrm{NMR}\left(126 \mathrm{MHz}\right.$, toluene- $\left.d_{8}, 80^{\circ} \mathrm{C}\right) \delta 294.0,216.8,159.7$, 153.3, 145.7, 138.8, 138.7, 137.8, 134.7 (br.s), 130.4, 130.1, 129.5, 129.1, 123.8 (q, ${ }^{1} J_{\mathrm{C}, \mathrm{F}}=293$ $\mathrm{Hz}), 123.4\left(\mathrm{q},{ }^{1} J_{\mathrm{C}, \mathrm{F}}=289 \mathrm{~Hz}\right), 122.4,122.3,113.4,85.3$ (hept, ${ }^{1} J_{\mathrm{C}, \mathrm{F}}=27 \mathrm{~Hz}$ ), 75.1, 58.0, 55.4, 54.8 (br.s), 53.0 (br.s), 22.3, 22.2, 22.1, 21.9; ${ }^{19} \mathrm{~F}$ NMR $\left(282 \mathrm{MHz}\right.$, toluene- $\left.d 8,60^{\circ} \mathrm{C}\right) \delta-65.89$, 70.73. Anal. Calcd for $\mathrm{C}_{33} \mathrm{H}_{36} \mathrm{Cl}_{2} \mathrm{~F}_{6} \mathrm{~N}_{2} \mathrm{O}_{3} \mathrm{Ru}(\%) \mathrm{C}, 49.88 ; \mathrm{H}, 4.57$; N, 3.53. Found: C, 49.91; $\mathrm{H}$, 4.52; N, 3.27. CCDC 1543695 contains the supplementary crystallographic data for this paper. 
These data can be obtained free of charge from The Cambridge Crystallographic Data Centre via http://www.ccdc.cam.ac.uk.

\section{References}

[1] S. Díez-González, $N$-Heterocyclic Carbenes: From laboratory curiosities to efficient synthetic tools, RSC Publishing, Cambridge, 2011.

[2] D. Bézier, J.-B. Sortais, C. Darcel, $N$-Heterocyclic carbene ligands and iron: an effective association for catalysis, Adv. Synth. Catal. 355 (2013) 19-33.

[3] C. Valente, S. Çalimsiz, K.H. Hoi, D. Mallik, M. Sayah, M.G. Organ, The development of bulky palladium NHC complexes for the most-challenging cross-coupling reactions, Angew. Chem., Int. Ed. 51 (2012) 3314-3332.

[4] G.C. Vougioukalakis, R.H. Grubbs, Ruthenium-based heterocyclic carbene-coordinated olefin metathesis catalysts, Chem. Rev. 110 (2010) 1746-1787.

[5] G.C. Fortman, S.P. Nolan, N-Heterocyclic carbene (NHC) ligands and palladium in homogeneous cross-coupling catalysis: a perfect union, Chem. Soc. Rev. 40 (2011) 5151-5169.

[6] S. Díez-González, N. Marion, S.P. Nolan, $N$-Heterocyclic carbenes in late transition metal catalysis, Chem. Rev. 109 (2009) 3612-3676.

[7] M.N. Hopkinson, C. Richter, M. Schedler, F. Glorius, An overview of N-heterocyclic carbenes, Nature 510 (2014) 485-496.

[8] Y. Chauvin, Olefin metathesis: the early days (Nobel lecture), Angew. Chem., Int. Ed. 45 (2006) 3740-3747.

[9] R.R. Schrock, Multiple metal-carbon bonds for catalytic metathesis reactions (Nobel lecture), Angew. Chem., Int. Ed. 45 (2006) 3748-3759.

[10] R.H. Grubbs, Olefin-metathesis catalysts for the preparation of molecules and materials (Nobel lecture), Angew. Chem., Int. Ed. 45 (2006) 3760-3765.

[11] R.H. Grubbs, Handbook of Metathesis, Wiley-VCH, Weinheim, 2003, Vols. 1-3.

[12] S.J. Connon, S. Blechert, Recent advances in alkene metathesis, in: C. Bruneau, P.H. Dixneuf (Eds.), Ruthenium catalysts and fine chemistry, Springer, Heidelberg, 2004, pp. 93-124.

[13] A. Fürstner, Olefin metathesis and beyond, Angew. Chem., Int. Ed. 39 (2000) 3012-3043.

[14] A.H. Hoveyda, A.R. Zhugralin, The remarkable metal-catalysed olefin metathesis reaction, Nature 450 (2007) 243-251.

[15] P.H. Deshmukh, S. Blechert, Alkene metathesis: the search for better catalysts, Dalton Trans. (2007) 2479-2491.

[16] D. Astruc, The metathesis reactions: from a historical perspective to recent developments,

New J. Chem. 29 (2005) 42-56.

[17] A.M. Lozano-Vila, S. Monsaert, A. Bajek, F. Verpoort, Ruthenium-based olefin metathesis catalysts derived from alkynes, Chem. Rev. 110 (2010) 4865-4909.

[18] A. Perfetto, C. Costabile, P. Longo, F. Grisi, Ruthenium olefin metathesis catalysts with frozen NHC ligand conformations, Organometallics 33 (2014) 2747-2759.

[19] K. Grela, Olefin Metathesis Theory and Practice, J. Wiley \& Sons: Hoboken, New Jersey, 2014.

[20] C. Samojłowicz, M. Bieniek, K. Grela, Ruthenium-based olefin metathesis catalysts bearing $N$-heterocyclic carbene ligands, Chem. Rev. 109 (2009) 3708-3742.

[21] C. Fischmeister, P.H. Dixneuf, New Ruthenium Catalysts for Alkene Metathesis, in: Y. Imamoglu, V. Dragutan (Eds.), Metathesis chemistry: From nanostructure design to synthesis of advanced materials, Springer, Dordrecht, 2007, pp. 3-27.

[22] M.R. Buchmeiser, Homogeneous metathesis polymerization by well-defined group VI and group VIII transition-metal alkylidenes: fundamentals and applications in the preparation of advanced materials, Chem. Rev. 100 (2000) 1565-1604. 
[23] A. Leitgeb, J. Wappel, C. Slugovc, The ROMP toolbox upgraded, Polymer 51 (2010) 29272946.

[24] I.C. Stewart, T. Ung, A.A. Pletnev, J.M. Berlin, R.H. Grubbs, Y. Schrodi, Highly efficient ruthenium catalysts for the formation of tetrasubstituted olefins via ring-closing metathesis, Org. Lett., 9 (2007) 1589-1592.

[25] J.M. Berlin, K. Campbell, T. Ritter, T.W. Funk, A. Chlenov, R.H. Grubbs, Rutheniumcatalyzed ring-closing metathesis to form tetrasubstituted olefins, Org. Lett. 9 (2007) 1339-1342.

[26] F. Grisi, A. Mariconda, C. Costabile, V. Bertolasi, P. Longo, Influence of syn and anti configurations of NHC backbone on Ru-catalyzed olefin metathesis, Organometallics 28 (2009) 4988-4995.

[27] C. Costabile, A. Mariconda, L. Cavallo, P. Longo, V. Bertolasi, F. Ragone, F. Grisi, The pivotal role of symmetry in the ruthenium-catalyzed ring-closing metathesis of olefins, Chem. Eur. J. 17 (2011) 8618-8629.

[28] Y. Borguet, G. Zaragoza, A. Demonceau, L. Delaude, Ruthenium catalysts bearing a benzimidazolylidene ligand for the metathetical ring-closure of tetrasubstituted cycloolefins, Dalton Trans. 44 (2015) 9744-9755.

[29] J. Tornatzky, A. Kannenberg, S. Blechert, New catalysts with unsymmetrical $N$-heterocyclic carbene ligands, Dalton Trans. 41 (2012) 8215-8225.

[30] H. Hiyama, Organofluorine compounds: Chemistry and applications, Springer, Berlin, 2000.

[31] R.D. Chambers, Fluorine in organic chemistry, Blackwell Publishing Ltd., Oxford, 2004.

[32] P. Kirsch, Modern fluoroorganic chemistry: Synthesis, reactivity, applications, second ed., Wiley-VCH, Weinheim, 2013.

[33] K. Uneyama, Organofluorine chemistry, Blackwell Publishing Ltd., Oxford, 2006.

[34] K. Müller, C. Faeh, F. Diederich, Fluorine in pharmaceuticals: looking beyond intuition, Science 317 (2007) 1881-1886.

[35] J. Wang, M. Sánchez-Roselló, J.L. Aceña, C. del Pozo, A.E. Sorochinsky, S. Fustero, V.A. Soloshonok, H. Liu, Fluorine in pharmaceutical industry: fluorine-containing drugs introduced to the market in the last decade (2001-2011), Chem. Rev. 114 (2014) 2432-2506.

[36] S. Purser, P.R. Moore, S. Swallow, V. Gouverneur, Fluorine in medicinal chemistry, Chem. Soc. Rev. 37 (2008) 320-330.

[37] I. Ojima, Fluorine in medicinal chemistry and chemical biology, Wiley-Blackwell, Chichester, 2009.

[38] R.E. Banks, B.E. Smart, J.C. Tatlow, Organofluorine chemistry: principles and commercial applications, Plenum Press, New York, 1994.

[39] F. Giornal, S. Pazenok, L. Rodefeld, N. Lui, J.-P. Vors, F.R. Leroux, Synthesis of diversely fluorinated pyrazoles as novel active agrochemical ingredients, J. Fluorine Chem. 152 (2013) 211.

[40] R. Berger, G. Resnati, P. Metrangolo, E. Weber, J. Hulliger, Organic fluorine compounds: a great opportunity for enhanced materials properties, Chem. Soc. Rev. 40 (2011) 3496-3508.

[41] R.C. da Costa, J.A. Gladysz, Fluorous phase-transfer activation of catalysts: application of a new rate-enhancement strategy to alkene metathesis, Chem. Commun. (2006) 2619-2621.

[42] R.C. da Costa, J.A. Gladysz, Syntheses and reactivity of analogues of Grubbs' second generation metathesis catalyst with fluorous phosphines: a new phase-transfer strategy for catalyst activation, Adv. Synth. Catal. 349 (2007) 243-254.

[43] R. Tuba, R. C. da Costa, H. S. Bazzi, J. A. Gladysz, Phase transfer activation of fluorous analogs of Grubbs' second-generation catalyst: ring-opening metathesis polymerization, ACS Catal. 2 (2012) 155-162. 
[44] R. Tuba, E.N. Brothers, J.H. Reibenspies, H.S. Bazzi, J.A. Gladysz, Crystal structure and computational investigation of an analogue of Grubbs' second generation catalyst with a fluorous phosphine, Inorg. Chem. 51 (2012) 9943-9949.

[45] M. Matsugi, D.P. Curran, Synthesis, reaction, and recycle of light fluorous Grubbs-Hoveyda catalysts for alkene metathesis, J. Org. Chem. 70 (2005) 1636-1642.

[46] S. Leach, C.J. Cordier, D. Morton, G.J. McKierman, S. Warriner, A. Nelson, A fluoroustagged linker from which small molecules are released by ring-closing metathesis, J. Org. Chem. 73 (2008) 2753-2759.

[47] D. Morton, S. Leach, C. Cordier, S. Warriner, A. Nelson, Synthesis of natural-product-like molecules with over eighty distinct scaffolds, Angew. Chem., Int. Ed. 48 (2009) 104-109.

[48] M. Matsugi, Y. Kobayashi, N. Suzumura, Y. Tsuchiya, T. Shioiri, Synthesis and RCM reactions using a recyclable Grubbs-Hoveyda metathesis catalyst activated by a light fluorous tag, J. Org. Chem. 75 (2010) 7905-7908.

[49] J. Kvíčala, M. Schindler, V. Kelbichová, M. Babuněk, M. Rybáčková, M. Kvíčalová, J. Cvačka, A. Březinová, Experimental and theoretical study of Hoveyda-Grubbs catalysts modified by perfluorohexyl ponytail in the alkoxybenzylidene ligand, J. Fluorine Chem. 153 (2013) 12-25. [50] M. Babuněk, O. Šimůnek, J. Hošek, M. Rybáčková, J. Cvačka, A. Březinová, J. Kvíčala, Heavy fluorous phosphine-free ruthenium catalysts for alkene metathesis, J. Fluorine Chem. 161 (2014) 66-75.

[51] F. Michalek, W. Bannwarth, Application of a Grubbs-Hoveyda metathesis catalyst noncovalently immobilized by fluorous-fluorous interactions, Helv. Chim. Acta 89 (2006) 10301037.

[52] V. Andrushko, D. Schwinn, C. C. Tzucke, F. Michalek, J. Horn, C. Mössner, W. Bannwarth, Tris(perfluoroalkyl)silyl entities as unexpectedly potent tags for the noncovalent immobilization of catalysts by fluorous-fluorous interactions: application to the synthesis of several perfluorotagged ligands, Helv. Chim. Acta 88 (2005) 936-949.

[53] J. Lim, S. S. Lee, J. Y. Ying, Silica-supported catalysts for ring-closing metathesis: effects of linker group and microenvironment on recyclability, Chem. Commun. (2008) 4312-4314.

[54] E. M. Hensle, J. Tobis, J. C. Tiller, W. Bannwarth, Ring-closing olefin metathesis in the aqueous phase of amphiphilic conetworks consisting of fluorophilic and hydrophilic compartments, J. Fluorine Chem. 129 (2008) 968-973.

[55] Q. Yao, Y. Zhang, Poly(fluoroalkyl acrylate)-bound ruthenium carbene complex: a fluorous and recyclable catalyst for ring-closing olefin metathesis, J. Am. Chem. Soc. 126 (2004) 74-75.

[56] Y. Kobayashi, S. Inukai, N. Kondo, T. Watanabe, Y. Sugiyama, H. Hamamoto, T. Shioiri, M. Matsugi, A medium fluorous Grubbs-Hoveyda 2nd generation catalyst for phase transfer catalysis of ring closing metathesis reactions, Tetrahedron Lett. 56 (2015) 1363-1366.

[57] J. Krause, O. Nuyken, K. Wurst, M.R. Buchmeiser, Synthesis and reactivity of homogeneous and heterogeneous ruthenium-based metathesis catalysts containing electron-withdrawing ligands, Chem. Eur. J. 10 (2004) 777-784.

[58] J. Krause, O. Nuyken, M.R. Buchmeiser, Factors relevant for the ruthenium-benzylidenecatalyzed cyclopolymerization of 1,6-heptadyines, Chem. Eur. J. 10 (2004) 2029-2035.

[59] L. Yang, M. Mayr, K. Wurst, M.R. Buchmeiser, Novel metathesis catalysts based on ruthenium 1,3-dimesityl-3,4,5,6-tetrahydropyrimidin-2-ylidenes: synthesis, structure, immobilization, and catalytic activity, Chem. Eur. J. 10 (2004) 5761-5770.

[60] T.S. Halbach, S. Mix, D. Fischer, S. Maechling, J.O. Krause, C. Sievers, S. Blechert, O. Nuyken, M.R. Buchmeiser, Novel ruthenium-based metathesis catalysts containing electronwithdrawing ligands: synthesis, immobilization, and reactivity, J. Org. Chem. 70 (2005) 46874694. 
[61] A.-F. Mingotaud, M. Krämer, C. Mingotaud, Catalytic surfactants for ring-opening metathesis polymerization and ring-closing metathesis in non-degassed micellar solutions, J. Mol. Catal. A 263 (2007) 39-47.

[62] J.C. Conrad, H.H. Parnas, J.L. Snelgrove, D.E. Fogg, Highly efficient Ru-pseudohalide catalysts for olefin metathesis, J. Am. Chem. Soc. 127 (2005) 11882-11883.

[63] S. Monfette, K.D. Camm, S.I. Gorelsky, D.E. Fogg, Electronic effects of the anionic ligand in ruthenium-catalyzed olefin metathesis, Organometallics 28 (2009) 944-946.

[64] S. Fustero, A. Simón-Fuentes, P. Barrio, G. Haufe, Olefin metathesis reactions with fluorinated substrates, catalysts, and solvents, Chem. Rev. 115 (2015) 871-930.

[65] V. Siano, I. d'Auria, F. Grisi, C. Costabile, P. Longo, Activity and stereoselectivity of Rubased catalyst bearing a fluorinated imidazolinium ligand, Cent. Eur. J. Chem. 9 (2011) 605-609.

[66] J. Hošek, M. Rybáčková, J. Čejka, J. Cvačka, J. Kvíčala, Synthesis of heavy fluorous ruthenium metathesis catalysts using the stereoselective addition of polyfluoroalkyllithium to sterically hindered diimines, Organometallics 34 (2015) 3327-3334.

[67] P. Lipovská, L. Rathouská, O. Šimůnek, J. Hošek, V. Kolaříková, M. Rybáčková, J. Cvačka, M. Svoboda, J. Kvíčala, Synthesis and catalytic activity of ruthenium complexes modified with chiral racemic per- and polyfluorooxaalkanoates, J. Fluorine Chem. 191 (2016) 14-22.

[68] A. Fürstner, L. Ackermann, B. Gabor, R. Goddard, C.W. Lehmann, R. Mynott, F. Stelzer, O. R. Thiel, Comparative investigation of ruthenium-based metathesis catalysts bearing $N$ heterocyclic carbene (NHC) ligands, Chem. Eur. J. 7 (2001) 3236-3253.

[69] T. Ritter, M.W. Day, R.H. Grubbs, Rate acceleration in olefin metathesis through a fluorineruthenium interaction, J. Am. Chem. Soc. 128 (2006) 11768-11769.

[70] D.R. Anderson, D.J. O'Leary, R.H. Grubbs, Ruthenium-olefin complexes: effect of ligand variation upon geometry, Chem. Eur. J. 14 (2008) 7536-7544.

[71] G.C. Vougioukalakis, R.H. Grubbs, Ruthenium olefin metathesis catalysts bearing an $N$ fluorophenyl- $N$-mesityl-substituted unsymmetrical $N$-heterocyclic carbene, Organometallics 26 (2007) 2469-2472.

[72] G.C. Vougioukalakis, R.H. Grubbs, Ruthenium-based olefin metathesis catalysts coordinated with unsymmetrical $N$-heterocyclic carbene ligands: synthesis, structure, and catalytic activity, Chem. Eur. J. 14 (2008) 7545-7556.

[73] C. K. Chung, R. H. Grubbs, Olefin metathesis catalyst: stabilization effect of backbone substitutions of N-heterocyclic carbene, Org. Lett. 10 (2008) 2693-2696.

[74] K. M. Kuhn, J.-B. Bourg, C. K. Chung, S. C. Virgil, R.H. Grubbs, Effects of NHC-backbone substitution on efficiency in ruthenium-based olefin metathesis, J. Am. Chem. Soc. 131 (2009) 5313-5320.

[75] A. Perfetto, C. Costabile, P. Longo, V. Bertolasi, F. Grisi, Probing the relevance of NHC ligand conformations in the Ru-catalysed ring-closing metathesis reaction, Chem. Eur. J. 19 (2013) 10492-10796.

[76] A. Perfetto, C. Costabile, P. Longo, F. Grisi, Ruthenium olefin metathesis catalysts with frozen NHC ligand conformations, Organometallics 33 (2014) 2747-2759.

[77] S.H. Hong, A. Chlenov, M.W. Day, R.H. Grubbs, Double C-H activation of an $N$-heterocyclic carbene ligand in a ruthenium olefin metathesis catalyst, Angew. Chem., Int. Ed. 46 (2007) 51485151.

[78] A. Perfetto, V. Bertolasi, C. Costabile, V. Paradiso, T. Caruso, P. Longo, F. Grisi, Methyl and phenyl substituent effects on the catalytic behavior of NHC ruthenium complexes, RSC Adv. 6 (2016) 95793-95804.

[79] D. Nelson, A. Collado, S. Manzini, S. Meiries, D.B. Cordes, A.M.Z. Slawin, S.P. Nolan, Methoxy-functionalized $N$-heterocyclic carbenes, Organometallics 33 (2014) 2048-2058. 
[80] S. Meiries, K. Speck, D.B. Cordes, A.M.Z. Slawin, S.P. Nolan, [Pd(IPr*OMe $)(\mathrm{acac}) \mathrm{Cl}]$ : Tuning the $N$-heterocyclic carbene in catalytic C-N bond formation, Organometallics 32 (2012) 330-339.

[81] G. Le Duc, S. Meiries, S.P. Nolan, Effect of electronic enrichment of NHCs on the catalytic activity of $[\mathrm{Pd}(\mathrm{NHC})(\mathrm{acac}) \mathrm{Cl}]$ in Buchwald-Hartwig coupling, Organometallics 32 (2013) 75477551.

[82] S. Leuthäußer, D. Schwarz, H. Plenio, Tuning the electronic properties of $N$-heterocyclic carbenes, Chem. Eur. J. 13 (2007) 7195-7203.

[83] S. Manzini, C.A. Urbina Blanco, D.J. Nelson, A. Poater, T. Lebl, S. Meiries, A.M.Z. Slawin, L. Falivene, L. Cavallo, S.P. Nolan, Evaluation of an olefin metathesis pre-catalyst with a bulky and electron-rich $N$-heterocyclic carbene, J. Organomet. Chem. 780 (2015) 43-48.

[84] S.N. Osipov, C. Bruneau, M. Picquet, A.F. Kolomiets, P.H. Dixneuf, Synthesis of fluorinecontaining cyclic amino acid derivatives via ring closing olefin metathesis, Chem. Commun. (1998) 2053-2054.

[85] S.N. Osipov, O.I. Artyushin, A.F. Kolomiets, C. Bruneau, P.H. Dixneuf, $\alpha-\mathrm{CF}_{3}-$ Substituted phosphorus containing analogs of $\alpha$-amino acids. Novel six- and seven-membered $\alpha$-amino phosphonates via ring closing metathesis with $\mathrm{LnRu}=\mathrm{C}=\mathrm{C}=\mathrm{CR}_{2}$ precatalyst, Synlett (2000) 10311033.

[86] S.N. Osipov, N.M. Kobelíkova, G.T. Shchetnikov, A.F. Kolomiets, C. Bruneau, P.H. Dixneuf, Novel synthesis of cyclic $\alpha$-amino acid esters via ene reaction and ruthenium-catalyzed ring rearrangement, Synlett (2001) 621-622.

[87] S.N. Osipov, O.I. Artyushin, A.F. Kolomiets, C. Bruneau, M. Picquet, P.H. Dixneuf, Synthesis of fluorine-containing cyclic $\alpha$-amino acid and $\alpha$-amino phosphonate derivatives by alkene metathesis, Eur. J. Org. Chem. (2001) 3891-3897.

[88] D. Semeril, J. Le Nôtre, C. Bruneau, P.H. Dixneuf, A.F. Kolomiets, S.N. Osipov, Fluorinecontaining $\alpha$-alkynyl amino esters and access to a new family of 3,4-dehydroproline analogues, New. J. Chem. 25 (2001) 16-18.

[89] M. Eckert, F. Monnier, G.T. Shchetnikov, I.D. Titanyuk, S.N. Osipov, L. Toupet, S. Dérien, P.H. Dixneuf, Tandem catalytic carbene addition/bicyclization of enynes. one-step synthesis of fluorinated bicyclic amino esters by ruthenium catalysis, Org. Lett. 7 (2005) 3741-3743.

[90] D.V. Vorobyeva, A.K. Mailyan, A.S. Peregudov, N.M. Karimova, T.P. Vasilyeva, I.S. Bushmarinov, C. Bruneau, P.H. Dixneuf, S.N. Osipov, Synthesis of functionalized CF3-containing heterocycles via [2,3]-sigmatropic rearrangement and sequential catalytic carbocyclization, Tetrahedron 67 (2011) 3524-3532.

[91] M. Eckert, S. Moulin, F. Monnier, I.D. Titanyuk, S.N. Osipov, T. Roisnel, S. Dérien, P.H. Dixneuf, Ruthenium-catalysed synthesis of fluorinated bicyclic amino esters through tandem carbene addition/cyclopropanation of enynes, Chem. Eur. J. 17 (2011) 9456-9462.

[92] A.K. Mailyan, I.M. Krylov, C. Bruneau, P.H. Dixneuf, S.N. Osipov, Access to cyclic $\alpha-\mathrm{CF}_{3}-$ substituted $\alpha$-amino acid derivatives by ring-closing metathesis of functionalized 1,7-enynes, Eur.

J. Org. Chem. (2013) 5353-5363.

[93] S.M. Masoud, A.K. Mailyan, V. Dorcet, T. Roisnel, P.H. Dixneuf, C. Bruneau, S.N. Osipov, Metathesis catalysts with fluorinated unsymmetrical NHC ligands, Organometallics 34 (2015) 2305-2313.

[94] P. Schwab, M.B. France, J.W. Ziller, R.H. Grubbs, A series of well-defined metathesis catalysts-synthesis of $\left[\mathrm{RuCl}_{2}\left(=\mathrm{CHR}^{\prime}\right)\left(\mathrm{PR}_{3}\right)_{2}\right]$ and its reactions, Angew. Chem., Int. Ed. Engl. 34 (1995) 2039-2041.

[95] J.S. Kingsbury, J.P.A. Harrity, B.L. Gray, A.H. Hoveyda, A recyclable Ru-based metathesis catalyst, J. Am. Chem. Soc. 121 (1999) 791-799. 


\section{Graphical abstract:}

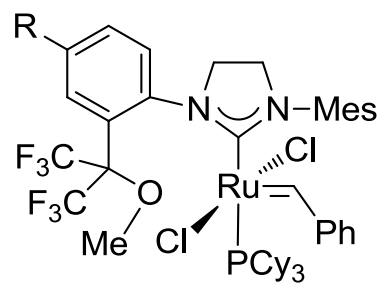

$\mathrm{R}=\mathrm{Me}, \mathrm{OMe}$

Grubbs-type catalyst

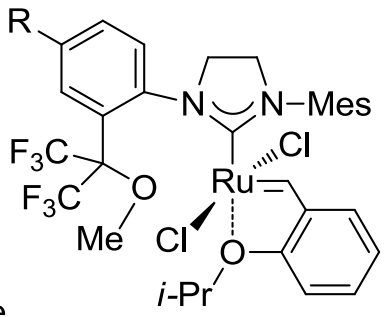

Hoveyda-type catalyst

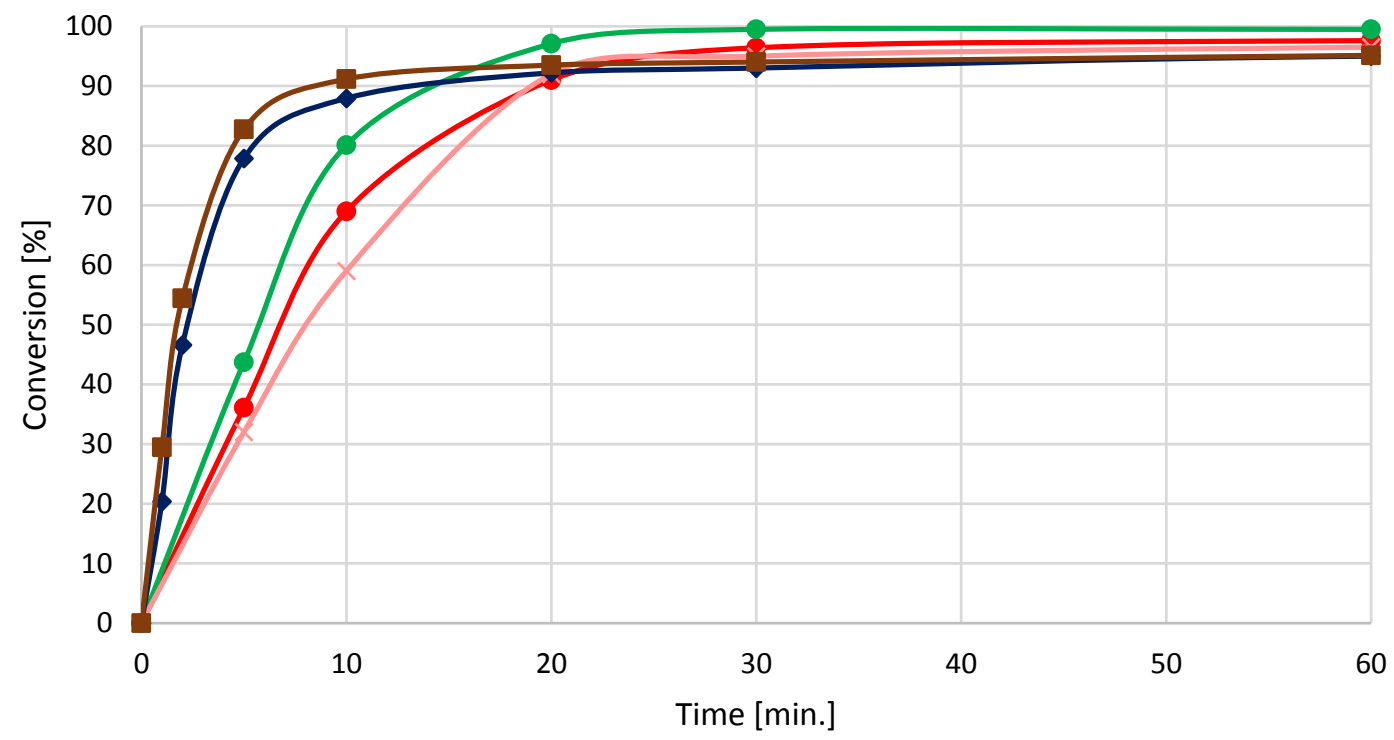

$\rightarrow$ G-II $\rightarrow \mathrm{H}-\mathrm{II} \leftarrow 1 \rightarrow 3 \rightarrow-4$

<InlineImage1>

Figure 4. RCM of DEDAM with catalysts 3, 4 as compared to 1, G-II and H-II
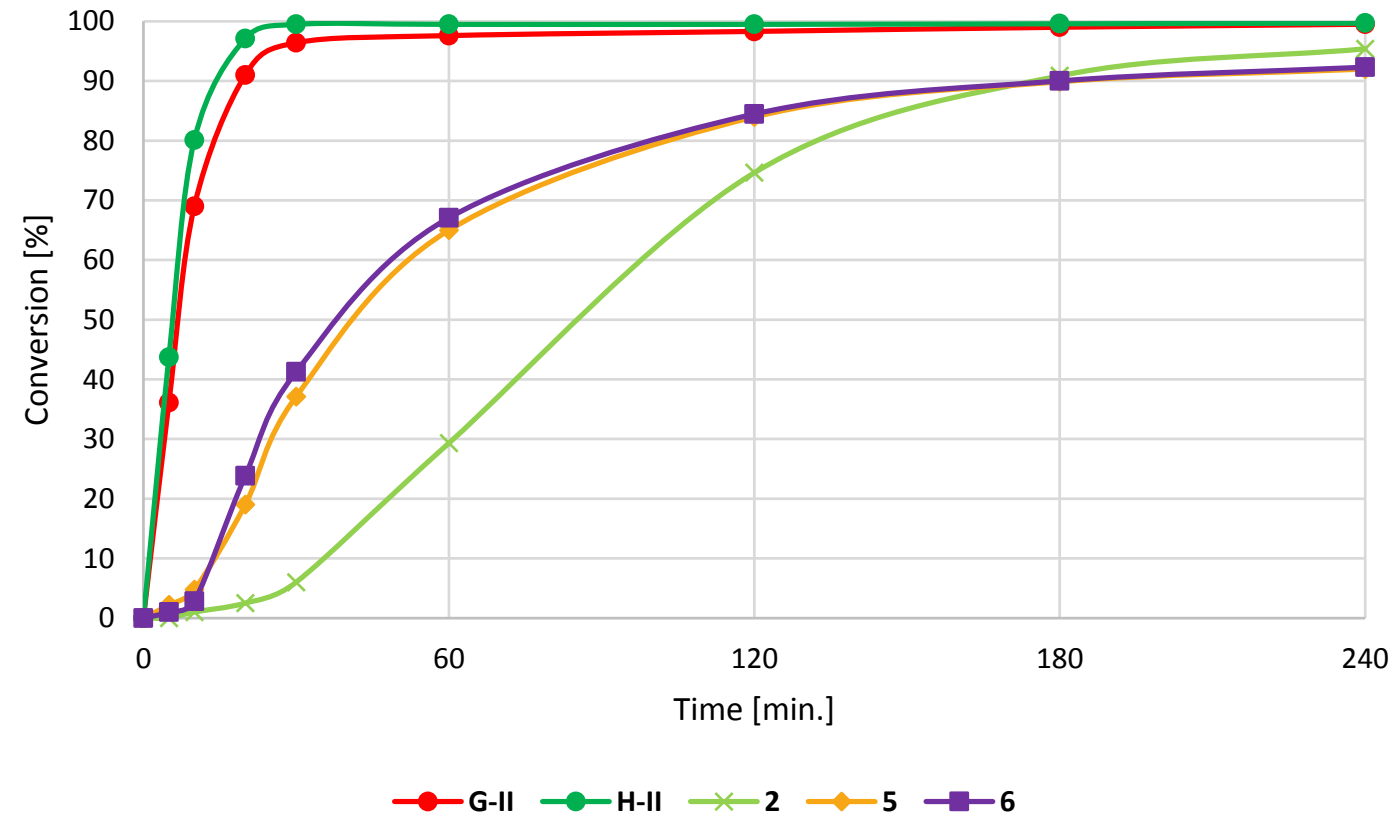

$<$ InlineImage2> 
Figure 5. RCM of DEDAM with catalysts 5, $\mathbf{6}$ as compared to 2, G-II and H-II
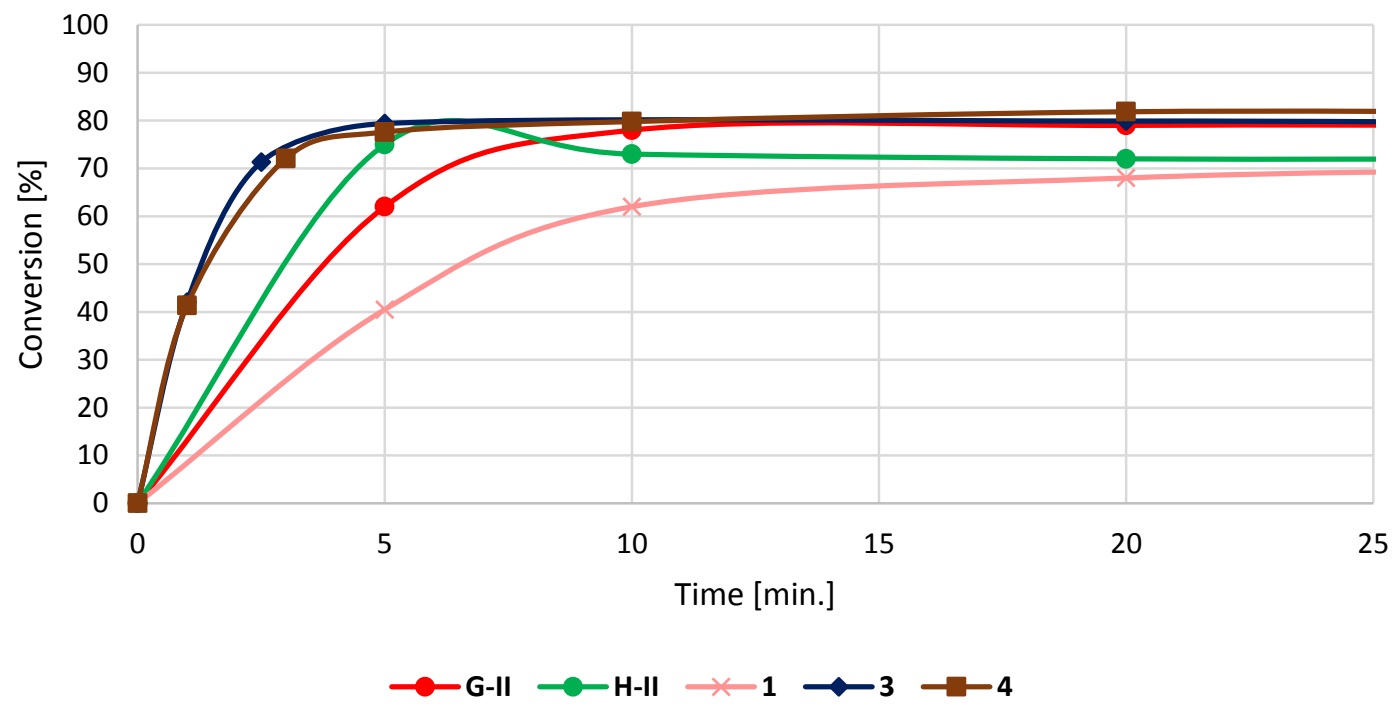

$<$ Inlinelmage3>

Figure 6. CM of allylbenzene with 1,3-diacetoxybut-2-ene with catalysts $\mathbf{3 , 4}$

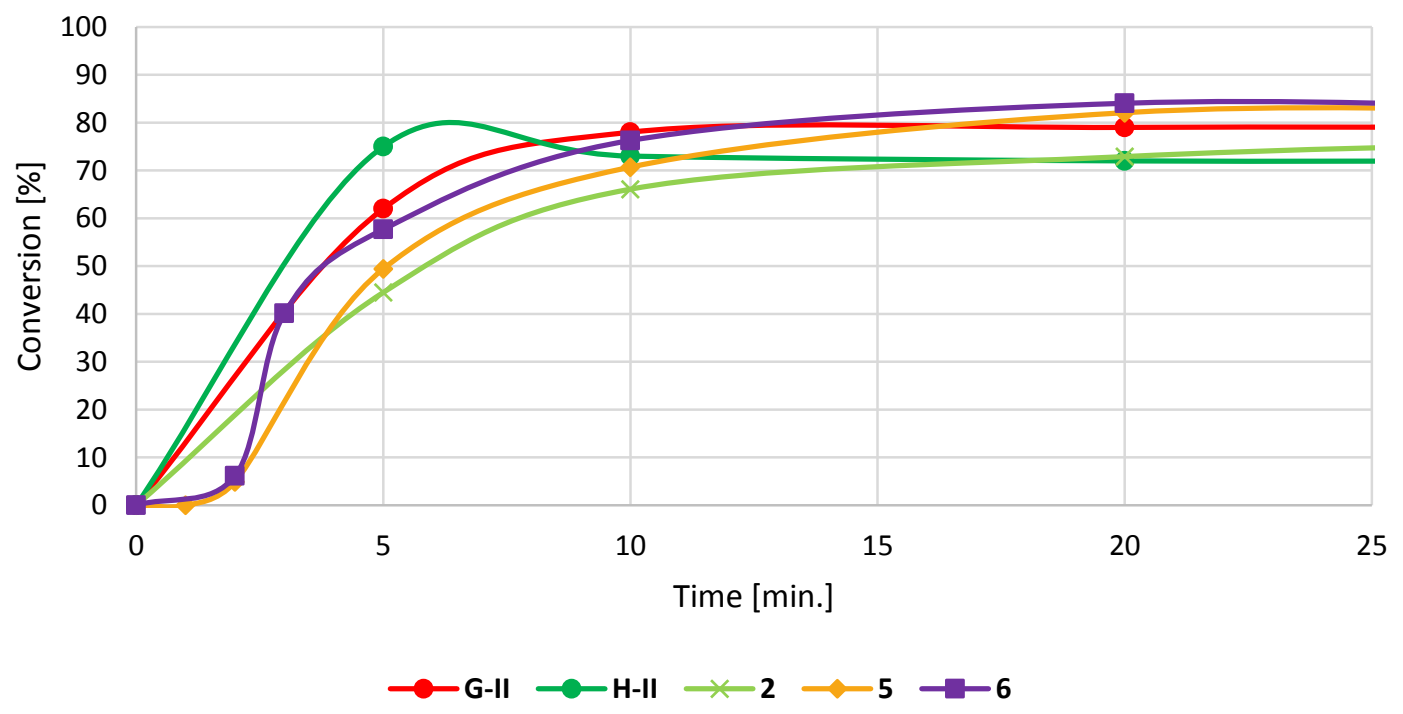

<InlineImage4>

Figure 7. CM of allylbenzene with 1,3-diacetoxybut-2-ene with catalysts 5, 6 